\title{
Selective Synthesis of $N$-Acylnortropane Derivatives in Palladium-Catalysed Aminocarbonylation
}

\author{
László Kollár 1,2,3 , Ádám Erdélyi ${ }^{1}$, Haroon Rasheed ${ }^{1}$ and Attila Takács 1,2,*D \\ 1 Department of Inorganic Chemistry, Faculty of Sciences, University of Pécs, Ifjúság útja 6, H-7624 Pécs, \\ Hungary; kollar@gamma.ttk.pte.hu (L.K.); erdelyi.adam99@gmail.com (Á.E.); \\ privateharoon@gmail.com (H.R.) \\ 2 János Szentágothai Research Centre, University of Pécs, Ifjúság útja 20, H-7624 Pécs, Hungary \\ 3 MTA-PTE Research Group for Selective Chemical Syntheses, Ifjúság útja 6, H-7624 Pécs, Hungary \\ * Correspondence: takacsattila@gamma.ttk.pte.hu; Tel.: +36-72-503-600
}

Citation: Kollár, L.; Erdélyi, Á.;

Rasheed, H.; Takács, A. Selective Synthesis of $N$-Acylnortropane Derivatives in Palladium-Catalysed Aminocarbonylation. Molecules 2021, 26, 1813. https://doi.org/10.3390/ molecules 26061813

Academic Editors: Julien Petrignet, Mauro Pineschi and Rafael Chinchilla

Received: 18 February 2021

Accepted: 18 March 2021

Published: 23 March 2021

Publisher's Note: MDPI stays neutral with regard to jurisdictional claims in published maps and institutional affiliations.

Copyright: (c) 2021 by the authors. Licensee MDPI, Basel, Switzerland. This article is an open access article distributed under the terms and conditions of the Creative Commons Attribution (CC BY) license (https:// creativecommons.org/licenses/by/ $4.0 /)$.

\begin{abstract}
The aminocarbonylation of various alkenyl and (hetero)aryl iodides was carried out using tropane-based amines of biological importance, such as 8-azabicyclo[3.2.1]octan-3-one (nortropinone) and $3 \alpha$-hydroxy-8-azabicyclo[3.2.1]octane (nortropine) as $N$-nucleophile. Using iodoalkenes, the two nucleophiles were selectively converted to the corresponding amide in the presence of $\mathrm{Pd}(\mathrm{OAc})_{2} / 2$ $\mathrm{PPh}_{3}$ catalysts. In the presence of several iodo(hetero)arenes, the application of the bidentate Xantphos was necessary to produce the target compounds selectively. The new carboxamides of varied structure, formed in palladium-catalyzed aminocarbonylation reactions, were isolated and fully characterized. In this way, a novel synthetic method has been developed for the producing of $\mathrm{N}$-acylnortropane derivatives of biological importance.
\end{abstract}

Keywords: aminocarbonylation; $N$-acylnortropanes; carbon monoxide; palladium

\section{Introduction}

Amide functionality can be found in numerous pharmaceutically, biologically active and agrochemical molecules [1-3], and its formation has been deeply studied [4,5]. There are several ways of introducing acyl functionality into simple model compounds or skeletons of practical importance. The application of conventional acylating agents is well known and is referenced in reviews [6], handbooks, and even textbooks [7]. Activated carboxy compounds are widely used acylating agents producing the desired amide derivative in the presence of amines. Acyl azides [8], anhydrides [9-11], as well as esters [12,13] can be successfully used in the formation of amide bond. The most efficient and widely-used method among the conventional amide synthesis is the reaction with acyl chlorides and amines [14-18]. Despite of the widespread use of acyl chlorides in amide synthesis, it has to be noted that acyl chlorides have significant limitations (e.g., hydrolysis, racemization, cleavage of protecting groups) [6]. Due to the importance of amides, several novel methods have also been developed for their synthesis [19-22].

The palladium-catalyzed aminocarbonylation is also an effective and widespread synthetic method for producing amides. Since the discovery of the aminocarbonylation by Heck et al. ("Heck-carbonylation") [23-25], the use of a primary or secondary amine, carbon monoxide, and aryl/alkenyl halides (or their synthetic surrogates, aryl triflates, and alkenyl triflates) in the presence of palladium catalysts leads to carboxamides in great variety [26-37]. Several $\alpha, \beta$-unsaturated carboxamides and aryl carboxamides, using palladium-catalyzed aminocarbonylation as a key reaction, were recently synthesized in our laboratory [38-45]. Just to illustrate the most recent developments, the functionalization of aromatic $N$-heterocycles in aminocarbonylation should be mentioned [46-49].

When the hundreds of examples of aminocarbonylation reactions are surveyed, we might conclude that great variety of aryl/alkenyl halides/triflates were investigated as 
substrates in the presence of simple primary or as secondary amines as $\mathrm{N}$-nucleophiles. However, a relatively small number of investigations are focused on the "opposite" reaction, i.e., applying amines of practical (for instance, pharmaceutical) importance and palladiumacyl intermediates formed in situ from relatively simple aryl or alkenyl halide by oxidative addition followed by carbon monoxide insertion. The palladium(II)-acyl species can be considered as highly reactive acylating agents [50-52].

Considering the fact that the palladium-catalyzed aminocarbonylation reactions have been widely applied to build-up amide functionality in several bioactive compounds and natural products, this method has been chosen to produce novel $\mathrm{N}$-acylnortropane derivatives in our research. The high selectivity, the mild reaction conditions, and the tolerance of wide range of functional groups make also more applicable the palladium-catalyzed aminocarbonylation for the synthesis of our target compounds than the conventional acylating methods.

The tropane-based derivatives have great importance in chemistry, which have been indicated by their wide-ranging use. The synthesis of $\mathrm{N}$-substituted nortropinones via reactivity umpolung of tropinone has been carried out by Willand et al. [53]. The role of twist-boat conformers in hydride reduction of tropinone has been investigated computationally and compared with experiments [54]. The diastereoselective acetylation of 6,7-dihydroxytropinones has been investigated in enzyme-catalyzed reaction [55]. Recently, a tropine-based ionic liquid was used for the resolution of racemic amino acids [56].

The functionalization of the tropane skeleton can be carried out efficiently in homogeneous catalytic reactions. The nonaflation-Heck reaction protocol was used for the synthesis of 1,3-tropadiene [57]. The palladium-catalyzed alkoxycarbonylation of the enoltriflate obtained from tropinone afforded the three epimers of diester in the presence of ethylene glycol [58]. The vinylboronate derivative obtained from tropinone underwent Suzuki-Miyaura coupling with aryl bromides resulting in aryl substituted nortopanes [59]. 3-Iodo-2-tropene was transformed to the corresponding 3-carboxamides and 3-esters in palladium-catalyzed amino- and alkoxycarbonylation, respectively [60]. A tropane-ring heterocyclic compound, scopolamine was synthesized in rhodium-catalyzed [4+3] cycloaddition reaction [61].

To the best of our knowledge, only just a few aromatic or $\alpha, \beta$-unsaturated $N$-acyl nortropane derivatives can be found in the literature $[62,63]$, and the using of nortropinone and nortropine as nucleophiles in aminocarbonylation has not been mentioned. It has to also be noted that the tropane-based derivatives have enormous biological relevance [64]. As a part of our long-standing interest in the investigation of the fine details of aminocarbonylation, we decided to investigate of use of tropane-derived nucleophiles in aminocarbonylation.

In this paper, the selective synthesis of $\mathrm{N}$-acylnortropane derivatives, possessing a variety of $\mathrm{N}$-acyl moieties will be described.

\section{Results}

\subsection{Aminocarbonylation of Iodoalkenes (1-5) in the Presence of Tropane-Based Nucleophiles $(\mathbf{a}, \mathbf{b})$}

Azabicyclo[3.2.1]octan-3-one (nortropinone) (a) and 3 $\alpha$-hydroxy-8-azabicyclo[3.2.1]oc tane (nortropine) (b) as $N$-nucleophiles were applied for the aminocarbonylation of iodoalke nes (such as 1-iodocyclohexene (1), 4-tert-butyl-1-iodocyclohexene (2), trans-1-iodo-1-octene (3), 17-iodoandrost-16-ene (4), and 2-iodobornene (5)) in the presence of palladium catalysts (Scheme 1). Based on the prior knowledge of our research group [65,66], a low-ligated, highly active $\mathrm{Pd}(0)$ catalyst, prepared in situ from $\mathrm{Pd}(\mathrm{OAc})_{2}$ and $\mathrm{PPh}_{3}$, was used. The reduction of $\mathrm{Pd}(\mathrm{II})$ to catalytically active, highly unsaturated $\mathrm{Pd}(0)$ species took place on the influence of the phosphine while it is oxidized to phosphine oxide [67-69]. 


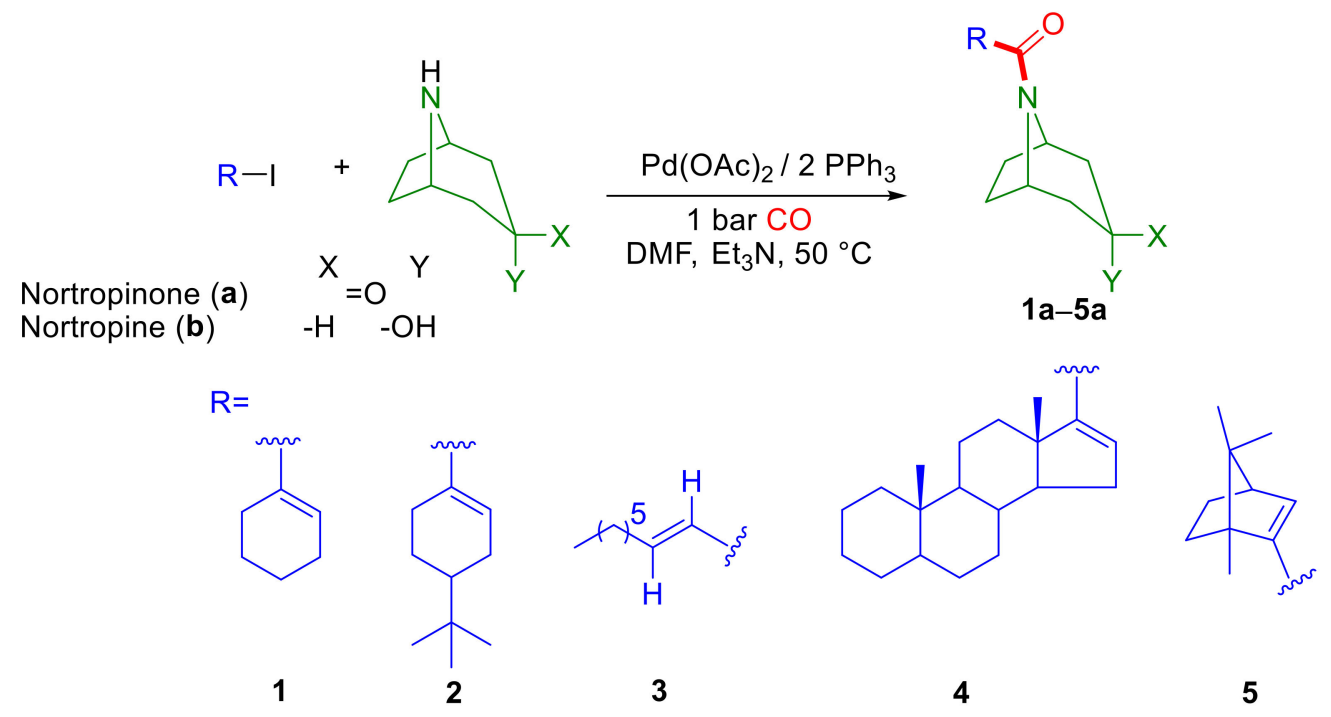

Scheme 1. Aminocarbonylation of tropane-based nucleophiles $(\mathbf{a}, \mathbf{b})$ in the presence of iodoalkenes (1-5) using $\mathrm{Pd}(\mathrm{OAc})_{2}+2 \mathrm{PPh}_{3}$ in situ catalysts.

The investigation of aminocarbonylation of nortropane-based nucleophiles was started with iodoalkenes (1-5), as shown on Scheme 1 and in Table 1. Nortropinone (a) was reacted with 1-iodocyclohexene (1) under atmospheric CO pressure. The target compound was observed just in traces by GC (entry 1) in short reaction time. Surprisingly, just $42 \%$ conversion was observed after $24 \mathrm{~h}$ (entry 2), and the complete conversion was not reached still after $48 \mathrm{~h}$ stirring (entry 3 ). Using elevated carbon monoxide pressure (40 bar), the substrate $\mathbf{1}$ was completely transformed to the corresponding carboxamide (1a) after $24 \mathrm{~h}$ (entry 5). It has to be noted, that double carbon monoxide insertion was not observed under these conditions. It can be stated, based on this experiment and our previous results, that the aminocarbonylation reactions of iodoalkenes do not provide the 2-ketocarboxamide type products [70,71]. In the presence of substrates 2 and $\mathbf{3}$, shorter reaction time (entries 6 and 7) was necessary to reach the complete conversion than in the case of 4 and 5 (entries 8 and 10). The $N$-acyl-nortropinone derivatives (1a-5a), synthesized in the aminocarbonylation reactions, were isolated in good yields (77-86\%).

The aminocarbonylation of nortropine (b) was also carried out in the presence of the above mentioned substrates (1-5). While the complete conversion of 17-iodoandrost-16-ene (4) to the corresponding carboxamide (4b) required $6 \mathrm{~h}$ (entry 15), the other substrates (1-3, 5) were fully converted in $1 \mathrm{~h}$ (entries 11-13,16).

Considering the behavior of the two nortropane-based nucleophiles, used in the reactions, decreased reactivity was observed in the case of nortropinone (a): the starting substrates (1-5) were completely converted into the target carboxamides in substantially longer reaction times $(4-24 \mathrm{~h}$ ). This phenomenon can be explained by the different conformation of the two nucleophiles. We believe that the different reactivity of the two secondary amines is due to steric factors. That is, the close to perfect chair conformation of nortropine is highly distorted when an $\mathrm{sp}^{2}$ carbon (that is, keto functionality) is introduced into the ring. This distortion could result in different reactivities both in the amine activation step toward the formation of the Pd-amide intermediate, and also in the product-forming step, where the acyl-amide-palladium(II) complex undergoes reductive elimination. 
Table 1. Aminocarbonylation of nortropane-based nucleophiles $(\mathbf{a}, \mathbf{b})$ in the presence of iodoalkenes (1-5) using $\mathrm{Pd}(\mathrm{OAc})_{2}+2 \mathrm{PPh}_{3}$ in situ catalyst ${ }^{\mathrm{a}}$.

\begin{tabular}{|c|c|c|c|c|c|}
\hline & $\begin{array}{l}\text { Alkenyl-I } \\
1-5 \\
\begin{array}{ll}\text { Nortropinone }(a) \\
\text { Nortropine }(\mathbf{b})\end{array} \quad-\mathrm{H}^{-}=\mathrm{O} \\
-\mathrm{OH}\end{array}$ & $\begin{array}{r}\mathrm{Pd}(\mathrm{OAc} \\
1 \mathrm{ba} \\
\mathrm{DMF}, \mathrm{Et}\end{array}$ & $\underset{50{ }^{\circ} \mathrm{C}}{\stackrel{2}{2 \mathrm{PPh}_{3}}}$ & $-5 a^{Y}$ & \\
\hline Entry & Substrate & Amine & $\begin{array}{l}\text { R. Time } \\
\text { [h] }\end{array}$ & $\begin{array}{c}\text { Conv. }^{\mathbf{b}} \\
{[18]}\end{array}$ & $\begin{array}{c}\text { Isolated } \\
\text { Yield }^{\mathrm{c}}[18]\end{array}$ \\
\hline 1 & 1-iodocyclohexene (1) & $\mathbf{a}$ & 4 & 3 & n.i. \\
\hline 2 & 1-iodocyclohexene (1) & $\mathbf{a}$ & 24 & 42 & n.i. \\
\hline 3 & 1-iodocyclohexene (1) & $\mathbf{a}$ & 48 & 88 & n.i. \\
\hline 4 & 1-iodocyclohexene (1) d & $\mathbf{a}$ & 4 & 70 & n.i. \\
\hline 5 & 1-iodocyclohexene (1) d & $\mathbf{a}$ & 24 & 100 & $80(\mathbf{1 a})$ \\
\hline 6 & 4-tert-butyl-1-iodocyclohexene (2) & $\mathbf{a}$ & 4 & 100 & $86(2 a)$ \\
\hline 7 & trans-1-iodo-1-octene (3) & $\mathbf{a}$ & 4 & 100 & $81(3 a)$ \\
\hline 8 & 17-iodoandrost-16-ene (4) & $\mathbf{a}$ & 24 & 100 & $80(4 a)$ \\
\hline 9 & 2-iodobornene (5) & a & 4 & 75 & n.i. \\
\hline 10 & 2-iodobornene (5) & $\mathbf{a}$ & 24 & 100 & $77(5 \mathbf{a})$ \\
\hline 11 & 1-iodocyclohexene (1) & b & 1 & 100 & 89 (1b) \\
\hline 12 & 4-tert-butyl-1-iodocyclohexene (2) & b & 1 & 100 & $79(\mathbf{2 b})$ \\
\hline 13 & trans-1-iodo-1-octene (3) & b & 1 & 100 & $65(3 b)$ \\
\hline 14 & 17-iodoandrost-16-ene (4) & b & 1 & 80 & n.i. \\
\hline 15 & 17-iodoandrost-16-ene (4) & b & 6 & 100 & $55(4 b)$ \\
\hline 16 & 2-iodobornene (5) & b & 1 & 100 & $63(5 b)$ \\
\hline
\end{tabular}

\subsection{Aminocarbonylation of Iodoarenes (6-10) in the Presence of Tropane-Based Nucleophiles $(\mathbf{a}, \mathbf{b})$}

After completing the reactions with the above mentioned iodoalkene substrates, we turned our attention to the investigation of aminocarbonylation of iodoarene compounds in the presence of nortropinone (a) and nortropine (b).

\subsubsection{Optimization Study with Iodobenzene (6)}

The aminocarbonylation of iodobenzene (6) was carried out in the presence of nortropin one (a) and nortropine (b) under different reaction conditions to find to optimal parameters for the further investigations (Scheme 2$). \mathrm{Pd}(\mathrm{OAc})_{2}$ was used as the catalyst precursor, and the influence of ligand, $\mathrm{CO}$ pressure, and temperature on the reactivity and selectivity was investigated.
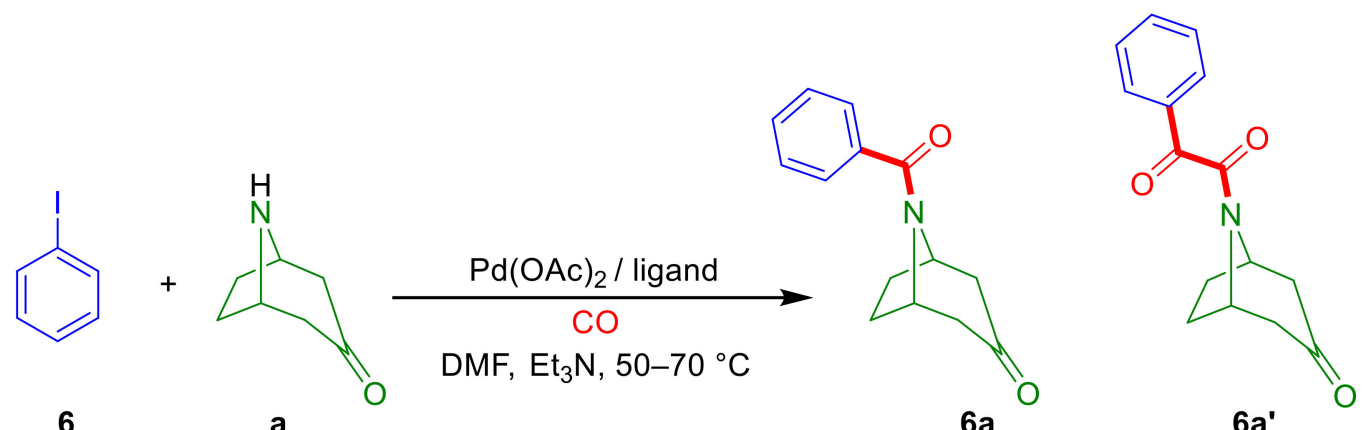

Scheme 2. Aminocarbonylation of iodobenzene (6) in the presence of nortropinone (a). 
Performing the reactions in the presence of $\mathrm{PPh}_{3}$ at 1 bar of $\mathrm{CO}$, it can be seen, that the conversion was very low both at 50 and $70^{\circ} \mathrm{C}$ (Figure 1). Using elevated carbon monoxide pressure (40 bar), 55\% conversion was detected after $24 \mathrm{~h}$. Under high pressure conditions, the corresponding 2-keto-carboxamide (6a') was also formed due to the second carbon monoxide insertion. The ratio of amide (6a): ketoamide (6a') type products was 56:44.

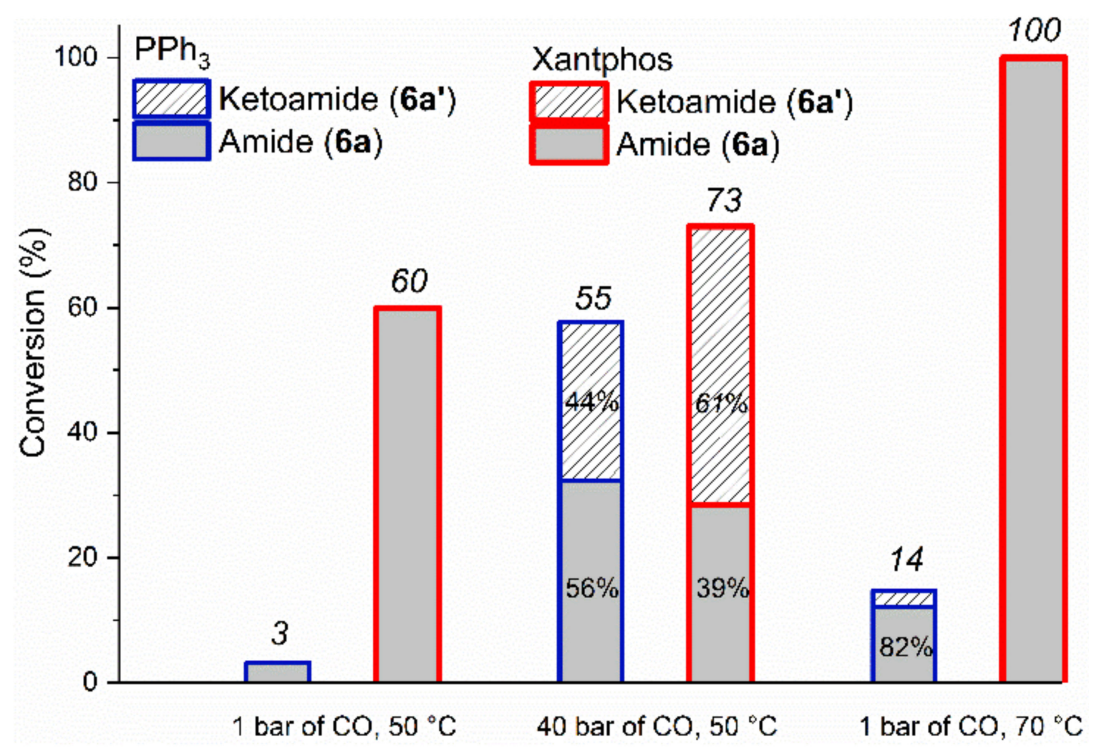

Figure 1. Conversion of $\mathbf{6}$ in the presence of a as a function of reaction conditions used during their aminocarbonylation in DMF after $24 \mathrm{~h}$.

Based on our previous results [72], the $\mathrm{PPh}_{3}$ was changed to the bidentate Xantphos to reach complete conversion in sort reaction time. Surprisingly, under atmospheric conditions, only $60 \%$ conversion was observed after $24 \mathrm{~h}$ in a selective reaction towards the corresponding carboxamide (6a). Higher conversion (73\%) was detected under elevated $\mathrm{CO}$ pressure (40 bar) and the ratio of $6 \mathbf{a}: 6 \mathbf{a}^{\prime}$ was 39:61. Increasing the temperature to $70{ }^{\circ} \mathrm{C}$, the target carboxamide (6a) was synthesized selectively after $6 \mathrm{~h}$ under atmospheric conditions (Figure 1).

The optimization study was also performed in the reaction on iodobenzene (6) with nortropine (b) under aminocarbonylation conditions (Scheme 3).<smiles>C=CC(=C)C(=O)C(=O)N1C2CCC1C(CC(=O)OC1CC3CCC(C1)N3C(=O)c1ccccc1)C(C)C(O)C2</smiles>

Scheme 3. Aminocarbonylation of iodobenzene (6) in the presence of nortropine (b).

Performing the reactions in the presence of $\mathrm{PPh}_{3}$ both under atmospheric and $40 \mathrm{bar}$ $\mathrm{CO}$ pressure, after $24 \mathrm{~h}$, the conversion was just $80 \%$ and $76 \%$, respectively. After $24 \mathrm{~h}$ stirring, the ratio of the amide (6b):ketoamide $\left(\mathbf{6 b}^{\prime}\right)$ type products was $66: 34$ under atmospheric CO pressure, while the chemoselectivity was shifted toward $\mathbf{6} \mathbf{b}^{\prime}$ at 40 bar (73\%). Changing the $\mathrm{PPh}_{3}$ to the bidentate Xantphos, as we expected, the iodobenzene was completely converted after $2 \mathrm{~h}$ at $50{ }^{\circ} \mathrm{C}$, producing the target carboxamide (6b) selectively 
(Figure 2). It has to be also mentioned, comparing the reactions of both nortropine-based nucleophile, that the nortropinone (b) showed higher reactivity than the nortropinone under same reaction conditions.

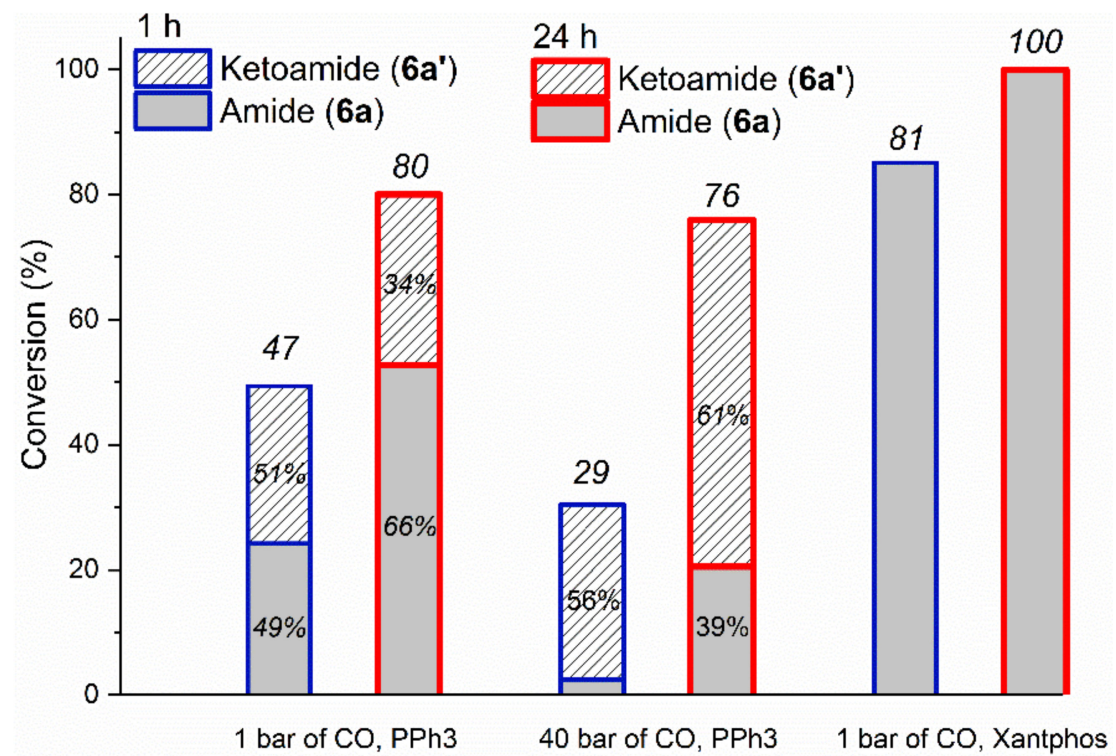

Figure 2. Conversion of $\mathbf{6}$ in the presence of $\mathbf{b}$ as a function of carbon monoxide pressure and the ligand used during the aminocarbonylation in DMF in $24 \mathrm{~h}$ stirring.

\subsubsection{Extending the Scope of Iodoarene Substrates}

According to the results of the paragraph in Section 2.1, it can be seen that the Xantphos could be a good choice to produce selectively $N$-acyl-nortropane derivatives selectively under mild aminocarbonylation conditions in the presence of other iodo-heteroaromatic substrates. In this way, 2-iodopyridine (7), 3-iodopyridine (8), 2-iodothiophene (9), and 5-iodoindole (10) were reacted with nortropinone (a) and nortropine (b) under aminocarbonylation conditions in the presence of $\mathrm{Pd}(\mathrm{OAc})_{2} /$ Xantphos catalysts (Scheme 4, Table 2).
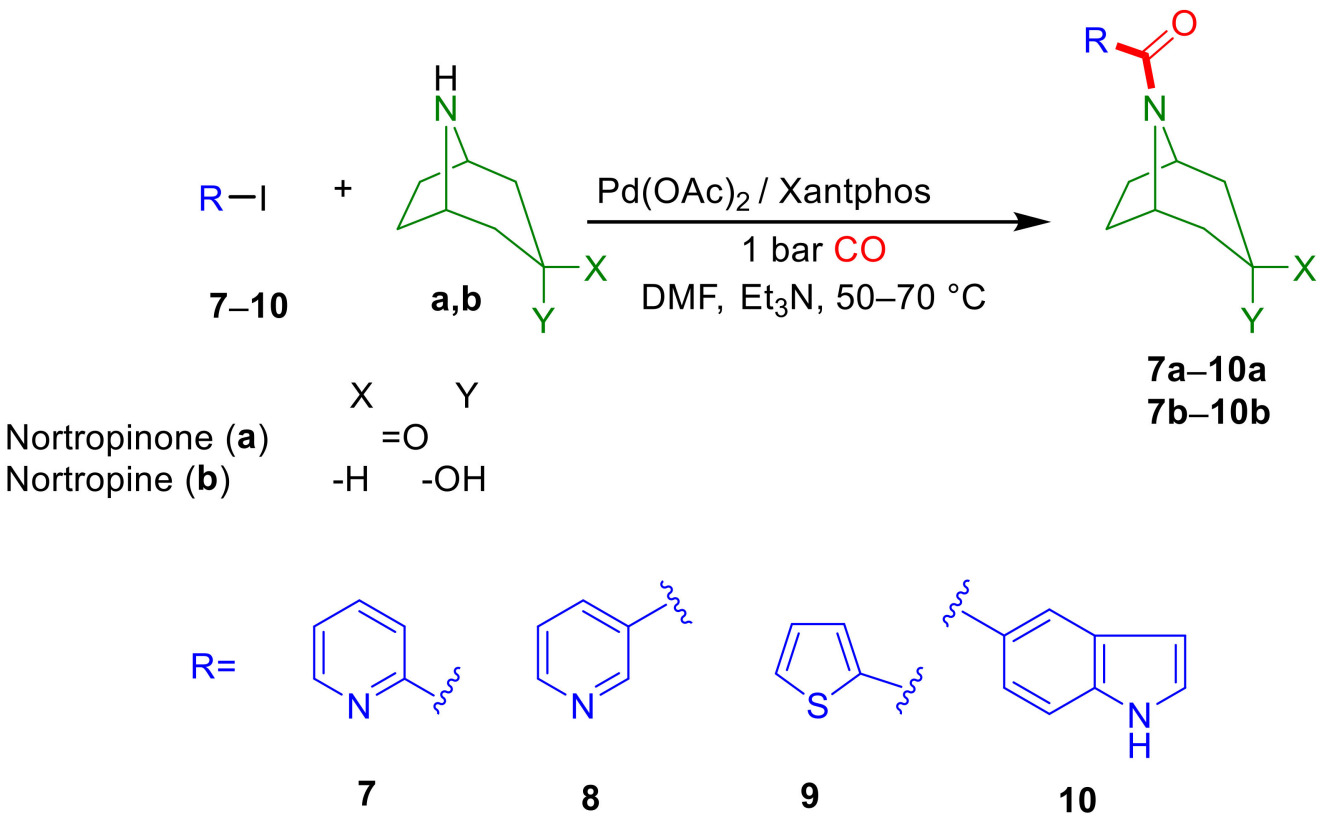

Scheme 4. Aminocarbonylation of iodo-heteroaromatic substrates (7-10) in the presence of nortropane-based nucleophiles $(\mathbf{a}, \mathbf{b})$. 
Table 2. Aminocarbonylation of nortropane-based nucleophiles $(\mathbf{a}, \mathbf{b})$ in the presence of iodoheteroaromatics (7-10) using $\mathrm{Pd}(\mathrm{OAc})_{2}+$ Xantphos in situ catalyst ${ }^{\mathrm{a}}$.

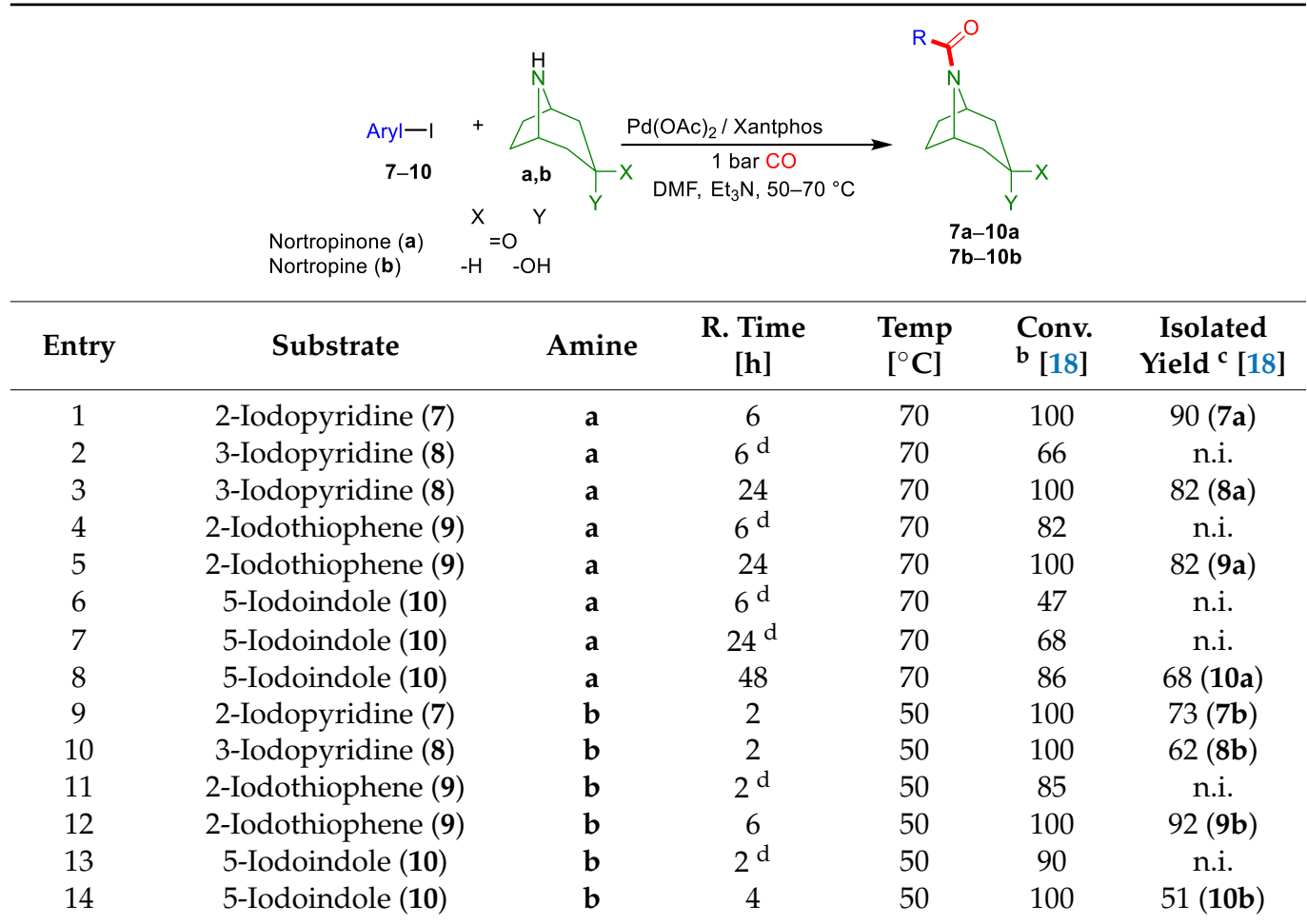

a Reaction conditions: $0.5 \mathrm{mmol}$ substrate (7-10), $0.55 \mathrm{mmol}$ of nucleophile $(\mathbf{a}, \mathbf{b}), 0.025 \mathrm{mmol}$ of Pd(OAc) $0.025 \mathrm{mmol}$ of Xantphos, $0.5 \mathrm{~mL}$ of $\mathrm{Et}_{3} \mathrm{~N}, 10 \mathrm{~mL}$ of DMF, 1 bar of CO. The reaction was purged with argon and then replaced by carbon monoxide. ${ }^{b}$ Determined by GC-MS. ${ }^{c}$ Yields of the isolated target compound (based on the substrates (7-10)); n.i. = not isolated. ${ }^{\mathrm{d}}$ Sample was taken and analyzed by GC.

Nitrogen-containing iodo-heteroarene substrates $(7,8)$ were converted completely into the correspondig carboxamides $(\mathbf{7 a}, \mathbf{8 a}, \mathbf{7 b}, \mathbf{8 b})$ after 2-6 h reaction time (entries $1,2,9,10)$. The difference between the reactivity of nortropinone (a) and nortropine (b) can easily be seen in the reactions with 2-iodothiophene (9) and 5-iodoinole (10). While in the presence of $\mathbf{b}$, the reaction was complete with substrates 9 and $\mathbf{1 0}$ in short reaction time (entries 12, $14)$; using nucleophile a, the reaction was conspicuously slower $(24-48 \mathrm{~h})$ (entries $5,7,8)$. The $\mathrm{N}$-acylnortropane derivatives synthesized in the reactions with substrates $\mathbf{7 - 1 0}$ were isolated in good to high yield (except 10b).

\section{Materials and Methods}

\subsection{General Procedures}

${ }^{1} \mathrm{H}$ and ${ }^{13} \mathrm{C}$ NMR spectra were recorded in $\mathrm{CDCl}_{3}$ or DMSO- $d_{6}$ on a Bruker Avance III 500 spectrometer (Bruker BioSpin Corp., Karlsruhe, Germany) at 500 and $125.7 \mathrm{MHz}$, respectively (See Supplementary Materials). Chemical shifts $\delta$ are reported in ppm relative to $\mathrm{CDCl}_{3}$ (7.26 and $77.00 \mathrm{ppm}$ for ${ }^{1} \mathrm{H}$ and ${ }^{13} \mathrm{C}$, respectively) or DMSO- $d_{6}$ (2.50 and $39.50 \mathrm{ppm}$ for ${ }^{1} \mathrm{H}$ and ${ }^{13} \mathrm{C}$, respectively). Elemental analyses were measured on a 1108 Carlo Erba apparatus (Carlo Erba Reagents SAS, Val de Reuil, France). The FT-IR spectra were taken in $\mathrm{KBr}$ pellets using a Nicolet IMPACT 400 spectrometer (Thermo Fisher Scientific, Waltham, MA, USA) applying a DTGS detector in the region of $400-4000 \mathrm{~cm}^{-1}$; the resolution was $4 \mathrm{~cm}^{-1}$. The amount of the samples was ca. $0.5 \mathrm{mg}$. Samples of the catalytic reactions were analysed with Chrom-Card Trace GC-Focus GC gas-chromatograph (Thermo Fisher Scientific, Waltham, MA, USA) fitted with a capillary column coated with OV-1 (injector temp. $250{ }^{\circ} \mathrm{C}$; oven: starting temp. $50{ }^{\circ} \mathrm{C}$ (hold-time $1 \mathrm{~min}$ ), heating rate $15^{\circ} \mathrm{C} \mathrm{min}-1$, final temp. $320^{\circ} \mathrm{C}$ (hold-time $11 \mathrm{~min}$ ); detector temp. $280^{\circ} \mathrm{C}$; carrier gas: helium (rate: $1 \mathrm{~mL} \mathrm{~min}^{-1}$ )). Mass spectrometry data have been obtained using a GC-MS system consisting of a Perkin 
Elmer AutoSystem XL gas-chromatograph (PerkinElmer Life and Analytical Sciences, Shelton, CT, USA). Melting points were measured with a Büchi apparatus (Büchi Labortechnik AG, Flawil, Switzerland). TLC plates (silica gel on TLC Al foils with fluorescence indicator $254 \mathrm{~nm}$ ) were purchased from Sigma-Aldrich (St. Louis, MO, USA). The eluents used in thin-layer chromatography are specified below.

The $\mathrm{Pd}(\mathrm{OAc})_{2}$, the ligands ( $\mathrm{PPh}_{3}$ and Xantphos), and the solvents were used without further purification. trans-1-Iodo-1-octene (3) and iodo-(hetero)aromatic substrates (610) were purchased from Sigma-Aldrich and were used without any further purification. The amine nucleophiles (nortropinone (a) and nortropine (b)) were purchased from TCI Chemicals and were used without further purification. 1-Iodocyclohexene (1) [73], 4-tertbutyl-1-iodocyclohexene (2) [73], 17-iodoandrost-16-ene (4) [74], and 2-iodobornene (5) were synthesized by the modified Barton-procedure [75,76]. The compound 6a is known, and the characterization data were in accordance with the literature. The $\mathbf{6 b}$ compound is also described, but its melting point is known only. ${ }^{1} \mathrm{H}$ and ${ }^{13} \mathrm{C}$ NMR data for these compounds are provided here for the sake of completeness.

3.2. Aminocarbonylation of Nortropane Derivatives $(\mathbf{a}, \mathbf{b})$ in the Presence of Iodoalkenes (1-5) and Iodoarenes (6-10) under Atmospheric Carbon Monoxide Pressure

In a typical experiment $\mathrm{Pd}(\mathrm{OAc})_{2}(5.6 \mathrm{mg}, 0.025 \mathrm{mmol})$, triphenylphosphine $(13.1 \mathrm{mg}$, $0.05 \mathrm{mmol}$ ) or Xantphos (14.5 mg, $0.025 \mathrm{mmol}$ ), iodoalkene (1-5) or iodo-(hetero)arene (6-11) substrates $(0.5 \mathrm{mmol})$, tropane-based nucleophiles (nortopinone (a), nortropine $(\mathbf{b}))$ $(0.55 \mathrm{mmol})$, and triethylamine $(0.5 \mathrm{~mL})$ were dissolved in DMF $(10 \mathrm{~mL})$ under argon in a $100 \mathrm{~mL}$ three-necked flask equipped with reflux condenser connected to a balloon filled with argon. The atmosphere was changed to carbon monoxide. The reaction was conducted for the given reaction time upon stirring at 50 or $70{ }^{\circ} \mathrm{C}$ and analyzed by GC and GC-MS. The cooled reaction mixture was then concentrated and evaporated to dryness under reduced pressure. The residue was dissolved in chloroform $(20 \mathrm{~mL})$ and washed twice with water $(20 \mathrm{~mL})$. The organic phase was dried over $\mathrm{Na}_{2} \mathrm{SO}_{4}$, filtered and evaporated under reduced pressure to a solid material. All compounds were subjected to column chromatography (Silicagel 60 (Sigma), $0.063-0.200 \mathrm{~mm}$ ), $\mathrm{CHCl}_{3} / \mathrm{MeOH}$ or $\mathrm{CHCl}_{3} /$ EtOAc eluent mixtures (the exact ratios are specified in Characterization (Section 3.4) for each compound).

3.3. Aminocarbonylation of Nortropane Derivatives $(\mathbf{a}, \mathbf{b})$ in the Presence of Iodoalkenes (1-5) and Iodoarenes (6-11) under High Carbon Monoxide Pressure

In a typical experiment $\mathrm{Pd}(\mathrm{OAc})_{2}$, triphenylphosphine or Xantphos, iodoalkene (1-5) or iodo-(hetero)arene (6-11) and tropane-based nucleophile $(\mathbf{a}, \mathbf{b})$ and triethylamine were used in the same amount as above and were dissolved in $10 \mathrm{~mL}$ of DMF under argon in a $100 \mathrm{~mL}$ autoclave. The atmosphere was changed to carbon monoxide, and the autoclave was pressurized to the given pressure with carbon monoxide (caution: high pressure carbon monoxide should only be used with adequate ventilation (hood) using CO sensors as well). The reaction was conducted for the given reaction time upon stirring at $50{ }^{\circ} \mathrm{C}$. After the given reaction time, the reaction mixture was cooled to room temperature, and the autoclave was carefully depressurized in a well-ventilated hood. The product mixture was analyzed by GC and GC-MS. The work-up of the reaction mixture was identical to that discussed for the atmospheric experiments.

\subsection{Characterization of the Products}

(1R,5S)-8-(Cyclohex-1-enecarbonyl)-8-azabicyclo[3.2.1]octan-3-one (1a), yield: $94 \mathrm{mg}$ (80\%); colourless oil; [Anal. Calcd. for $\mathrm{C}_{14} \mathrm{H}_{19} \mathrm{NO}_{2}$ : C, 72.07; $\mathrm{H}, 8.21 ; \mathrm{N}, 6.0$. Found: C, 71.87; $\mathrm{H}, 8.16 ; \mathrm{N}, 6.10] ; R_{\mathrm{f}}\left(30 \% \mathrm{EtOAc}, 70 \% \mathrm{CHCl}_{3}\right) 0.5 ;{ }^{1} \mathrm{H} \mathrm{NMR}\left(500 \mathrm{MHz}, \mathrm{CDCl}_{3}\right): 5.99$ $(1 \mathrm{H}, \mathrm{s}, \mathrm{C}=\mathrm{CH}), 4.69(2 \mathrm{H}, \mathrm{br} \mathrm{s}, \mathrm{N}-\mathrm{CH}), 2.62\left(2 \mathrm{H}, \mathrm{br} \mathrm{s}, \mathrm{CH}-\mathrm{CH}_{a} \mathrm{H}_{\mathrm{b}}\right), 2.37(2 \mathrm{H}, \mathrm{d}, 16 \mathrm{~Hz}$, $\left.\mathrm{C}(\mathrm{O})-\mathrm{CH}_{\mathrm{a}} \mathrm{H}_{b}\right), 2.27-2.18\left(2 \mathrm{H}, \mathrm{m}\right.$, cyclohex. $\left.\mathrm{CH}_{2}\right), 2.14-2.07\left(2 \mathrm{H}\right.$, br s, $\left.\mathrm{CH}-\mathrm{CH}_{\mathrm{a}} \mathrm{H}_{b}\right), 2.06-2.00$ $\left(2 \mathrm{H}, \mathrm{m}\right.$, cyclohex. $\left.\mathrm{CH}_{2}\right), 1.73-1.64\left(4 \mathrm{H}, \mathrm{m}\right.$, overlapping of cyclohex. $\mathrm{CH}_{2}$ and $\left.\mathrm{C}(\mathrm{O})-\mathrm{CH}_{a} \mathrm{H}_{\mathrm{b}}\right)$, 1.63-1.57 (2 H, m cyclohex. $\left.\mathrm{CH}_{2}\right) ;{ }^{13} \mathrm{C}$ NMR $\left(125 \mathrm{MHz}, \mathrm{CDCl}_{3}\right): 207.7,170.2,134.5,129.4$, 54.8 (br s, 2 C), 49.4 (br s, 2 C), 28.9 (br s, 2 C), 25.8, 24.7, 22.0, 21.6. MS m/z (rel. int.): 233 
(26, [M] $\left.]^{+}\right), 205$ (6), 176 (7), 148 (3), 124 (19), 109 (100), 81 (67), 53 (27). IR (KBr, v ( $\left.\left.\mathrm{cm}^{-1}\right)\right)$ : 1716 (CO), 1655 (C=C), 1628 (Amide I.), 1413.

(1R,5S)-8-(4-(tert-Butyl)cyclohex-1-enecarbonyl)-8-azabicyclo[3.2.1]octan-3-one (2a) (diastereomers can be distinguished), yield: $125 \mathrm{mg}$ (86\%); white solid, m.p. 123-124 ${ }^{\circ} \mathrm{C}$, [Anal. Calcd. for $\mathrm{C}_{18} \mathrm{H}_{27} \mathrm{NO}_{2}$ : C, 74.70; $\mathrm{H}, 9.40 ; \mathrm{N}, 4.80$. Found: $\left.\mathrm{C}, 74.97 ; \mathrm{H}, 9.44 ; \mathrm{N}, 4.93\right] ; \mathrm{R}_{\mathrm{f}}(10 \%$ EtOAc, $\left.90 \% \mathrm{CHCl}_{3}\right) 0.66 ;{ }^{1} \mathrm{H} \mathrm{NMR}\left(500 \mathrm{MHz}, \mathrm{CDCl}_{3}\right): 7.11-7.07 / 6.08-6.02(1 \mathrm{H}, \mathrm{m}, \mathrm{C}=\mathrm{CH})$, $4.78(2 \mathrm{H}, \mathrm{br} \mathrm{s}, \mathrm{N}-\mathrm{CH}), 2.68\left(2 \mathrm{H}, \mathrm{br} \mathrm{s}, \mathrm{CH}-\mathrm{CH}_{a} \mathrm{H}_{\mathrm{b}}\right), 2.51\left(1 \mathrm{H}, \mathrm{m}, \mathrm{CH}-\mathrm{C}\left(\mathrm{CH}_{3}\right)_{3}\right), 2.42(2 \mathrm{H}, \mathrm{d}$, $\left.16 \mathrm{~Hz}, \mathrm{C}(\mathrm{O})-\mathrm{CH}_{\mathrm{a}} \mathrm{H}_{b}\right), 2.32-2.20\left(2 \mathrm{H}, \mathrm{m}\right.$, cyclohex. $\left.\mathrm{CH}_{2}\right), 2.13-2.06\left(2 \mathrm{H}, \mathrm{br} \mathrm{s}, \mathrm{CH}-\mathrm{CH}_{\mathrm{a}} \mathrm{H}_{b}\right)$, 1.98-1.87 $\left(2 \mathrm{H}, \mathrm{m}\right.$, cyclohex. $\left.\mathrm{CH}_{2}\right), 1.80-1.68\left(2 \mathrm{H}, \mathrm{m}, \mathrm{C}(\mathrm{O})-\mathrm{CH}_{a} \mathrm{H}_{\mathrm{b}}\right), 1.41-1.23(2 \mathrm{H}, \mathrm{m}$, cyclohex. $\left.\mathrm{CH}_{2}\right), 0.89\left(9 \mathrm{H}, \mathrm{s}, \mathrm{C}\left(\mathrm{CH}_{3}\right)_{3}\right) ;{ }^{13} \mathrm{C} \mathrm{NMR}\left(125 \mathrm{MHz}, \mathrm{CDCl}_{3}\right): 207.7,170.9 / 170.1$, 140.9/134.3, 129.9/129.8, 55.3 (br s, 2 C), 49.5 (br s, 2 C), 43.5/43.2, 32.2/32.1, 28.7 (br s, 2 C), 27.7/27.5, 27.1, 26.6/26.5, 23.6/23.5. MS m/z (rel. int.): 289 (52, [M] $\left.{ }^{+}\right), 274(10), 261$ (6), 232 (24), 204 (8), 165 (100), 124 (40), 81 (39), 57 (53). IR (KBr, v ( $\left.\left.\mathrm{cm}^{-1}\right)\right)$ : 1714 (CO), 1663 (C=C), 1618 (Amide I.), 1410.

(1R,5S)-8-((E)-Non-2-enoyl)-8-azabicyclo[3.2.1]octan-3-one (3a), yield: $107 \mathrm{mg}(81 \%)$; colourless oil, [Anal. Calcd. for $\mathrm{C}_{16} \mathrm{H}_{25} \mathrm{NO}_{2}$ : $\mathrm{C}, 72.97 ; \mathrm{H}, 9.57 ; \mathrm{N}, 5.32$. Found: $\mathrm{C}, 72.76 ; \mathrm{H}, 9.44$; $\mathrm{N}, 5.48] ; R_{\mathrm{f}}\left(10 \% \mathrm{EtOAc}, 90 \% \mathrm{CHCl}_{3}\right) 0.66 ;{ }^{1} \mathrm{H} \mathrm{NMR}\left(500 \mathrm{MHz}, \mathrm{CDCl}_{3}\right): 7.03(1 \mathrm{H}, \mathrm{dt}, J$ $15.0 \mathrm{~Hz}, 7.0 \mathrm{~Hz},=\mathrm{CH}), 6.23(1 \mathrm{H}, \mathrm{dt}, J 15.0 \mathrm{~Hz}, 1.4 \mathrm{~Hz},=\mathrm{CH}), 4.97(1 \mathrm{H}, \mathrm{br} \mathrm{s}, \mathrm{N}-\mathrm{CH}), 4.60(1$ $\mathrm{H}$, br s, N-CH), 2.85-2.75 (1 H, m, CH-CH $\left.\mathrm{H}_{\mathrm{b}}\right), 2.60-2.51\left(1 \mathrm{H}, \mathrm{m}, \mathrm{CH}-\mathrm{CH}_{a} \mathrm{H}_{\mathrm{b}}\right), 2.49-2.36$ $\left(2 \mathrm{H}, \mathrm{m}, \mathrm{C}(\mathrm{O})-\mathrm{CH}_{\mathrm{a}} \mathrm{H}_{b}\right), 2.28-2.14\left(3 \mathrm{H}, \mathrm{m}\right.$, overlapping of $\mathrm{CH}-\mathrm{CH}_{\mathrm{a}} \mathrm{H}_{b}$ and $\left.=\mathrm{CH}-\mathrm{CH}_{2}\right), 2.09$ $\left(1 \mathrm{H}\right.$, br s, $\left.\mathrm{CH}-\mathrm{CH}_{\mathrm{a}} \mathrm{H}_{b}\right), 1.87-1.77\left(1 \mathrm{H}, \mathrm{m}, \mathrm{C}(\mathrm{O})-\mathrm{CH}_{a} \mathrm{H}_{\mathrm{b}}\right), 1.75-1.65\left(1 \mathrm{H}, \mathrm{m}, \mathrm{C}(\mathrm{O})-\mathrm{CH}_{a} \mathrm{H}_{\mathrm{b}}\right)$, 1.52-1.42 (2 H, m, $\left.\mathrm{CH}_{2} \mathrm{CH}_{3}\right), 1.38-1.21\left(6 \mathrm{H}, \mathrm{br} \mathrm{m}, 3 \times \mathrm{CH}_{2}\right), 0.89\left(3 \mathrm{H}, \mathrm{t}, 7.2 \mathrm{~Hz}, \mathrm{CH}_{2} \mathrm{CH}_{3}\right)$; ${ }^{13} \mathrm{C}$ NMR $\left(125 \mathrm{MHz}, \mathrm{CDCl}_{3}\right): 207.5,163.7,148.1,119.9,53.6,51.4,50.01,48.8,32.5,31.6,29.9$, 28.9, 28.3, 27.8, 22.5, 14.0. MS m/z (rel. int.): 263 (9, [M] $\left.{ }^{+}\right), 235$ (1), 206 (10), 178 (7), 150 (11), 124 (73), 81 (15), 55 (100). IR (KBr, $v\left(\mathrm{~cm}^{-1}\right)$ ): 1718 (CO), 1660 (C=C.), 1619 (Amide I.), 1425.

(1R,5S)-8-((10S,13S)-10,13-dimethyl-2,3,4,5,6,7,8,9,10,11,12,13,14,15-tetradecahydro-1H-cyclopen ta[a]phenanthrene-17-carbonyl)-8-azabicyclo[3.2.1]octan-3-one (4a), yield: $163 \mathrm{mg}(80 \%)$; pale white solid, m.p. ${ }^{186}-187^{\circ} \mathrm{C}$, [Anal. Calcd. for $\mathrm{C}_{27} \mathrm{H}_{39} \mathrm{NO}_{2}$ : C, 79.17; $\mathrm{H}, 9.60 ; \mathrm{N}, 3.42$. Found: C, 79.13; $\mathrm{H}, 9.82 ; \mathrm{N}, 3.45] ; \mathrm{R}_{\mathrm{f}}\left(20 \% \mathrm{EtOAc}, 80 \% \mathrm{CHCl}_{3}\right) 0.5 ;{ }^{1} \mathrm{H}$ NMR $(500 \mathrm{MHz}$, $\left.\mathrm{CDCl}_{3}\right)$ : $6.00(1 \mathrm{H}, \mathrm{s},=\mathrm{CH}), 5.09 / 4.89\left(1 \mathrm{H}, \mathrm{br} \mathrm{s}, \mathrm{N}-\mathrm{CH}_{2}\right), 4.62 / 3.56\left(1 \mathrm{H}, \mathrm{br} \mathrm{s}, \mathrm{N}-\mathrm{CH}_{2}\right), 2.69$ $\left(2 \mathrm{H}, \mathrm{br} \mathrm{s}, \mathrm{CH}-\mathrm{CH}_{\mathrm{a}} \mathrm{H}_{\mathrm{b}}\right), 2.43\left(2 \mathrm{H}, \mathrm{d}, 16.1 \mathrm{~Hz}, \mathrm{C}(\mathrm{O})-\mathrm{CH}_{\mathrm{a}} \mathrm{H}_{\mathrm{b}}\right), 2.33-2.22(1 \mathrm{H}, \mathrm{m}$, steroid$\mathrm{CH}), 2.15-2.05\left(2 \mathrm{H}\right.$, br s, $\left.\mathrm{CH}-\mathrm{CH}_{\mathrm{a}} \mathrm{H}_{\mathrm{b}}\right), 2.01-1.92(1 \mathrm{H}, \mathrm{m}$, steroid-CH $), 1.84-0.75(22 \mathrm{H}$, $\mathrm{C}(\mathrm{O})-\mathrm{CH}_{\mathrm{a}} \mathrm{H}_{\mathrm{b}}$ and 22 steroidal skeleton $\left.\mathrm{H}\right), 1.11\left(3 \mathrm{H}, \mathrm{s}, \mathrm{CH}_{3}\right), 0.85\left(3 \mathrm{H}, \mathrm{s}, \mathrm{CH}_{3}\right) ;{ }^{13} \mathrm{C} \mathrm{NMR}$ (125 MHz, $\mathrm{CDCl}_{3}$ ): 207.8, 166.3, 149.7, 132.3, 56.8, 55.3, 54.9, 49.8, 48.6, 47.3, 38.5, 36.5, 34.8, $33.9,32.2,32.1,29.6,29.0,28.9,26.8,22.1,20.6,16.8,12.2$. MS m/z (rel. int.): 409 (100, [M] ${ }^{+}$), 394 (95), 366 (8), 285 (46), 206 (4), 161 (8), 105 (14), 67 (29). IR (KBr, v ( $\left.\left.\mathrm{cm}^{-1}\right)\right): 1731$ (CO), 1635 (C=C), 1599 (Amide I.), 1420.

(1R,5S)-8-((1S,4R)-1,7,7-trimethylbicyclo[2.2.1]hept-2-ene-2-carbonyl)-8-azabicyclo[3.2.1]octan-3one (5a), yield: $111 \mathrm{mg}(77 \%)$; grey solid, m.p. $126-127^{\circ} \mathrm{C}$, [Anal. Calcd. for $\mathrm{C}_{18} \mathrm{H}_{25} \mathrm{NO}_{2}: \mathrm{C}$, 75.22; $\mathrm{H}, 8.77 ; \mathrm{N}, 4.87$. Found: $\mathrm{C}, 74.61 ; \mathrm{H}, 9.02 ; \mathrm{N}, 5.10] ; R_{\mathrm{f}}\left(20 \% \mathrm{EtOAc}, 80 \% \mathrm{CHCl}_{3}\right) 0.5$; ${ }^{1} \mathrm{H} \mathrm{NMR}\left(500 \mathrm{MHz}, \mathrm{CDCl}_{3}\right): 6.22(1 \mathrm{H}, \mathrm{s},=\mathrm{CH}), 5.11 / 4.85\left(1 \mathrm{H}, \mathrm{br} \mathrm{s}, \mathrm{N}-\mathrm{CH}_{2}\right), 4.78 / 3.56(1 \mathrm{H}$, br s, N-CH $), 2.76\left(2 \mathrm{H}, \mathrm{br} \mathrm{s}, \mathrm{CH}-\mathrm{CH}_{a} \mathrm{H}_{\mathrm{b}}\right), 2.51(1 \mathrm{H}, \mathrm{dd}, 4.0 \mathrm{~Hz}, 3.6 \mathrm{~Hz}, 4-\mathrm{CH}), 2.46(2 \mathrm{H}, \mathrm{br}$ $\left.\mathrm{s}, \mathrm{C}(\mathrm{O})-\mathrm{CH}_{\mathrm{a}} \mathrm{H}_{b}\right), 2.08\left(2 \mathrm{H}, \mathrm{br} \mathrm{s}, \mathrm{CH}-\mathrm{CH}_{\mathrm{a}} \mathrm{H}_{b}\right), 2.02-1.92\left(1 \mathrm{H}, \mathrm{m}, 5-\mathrm{CH}_{\mathrm{c}} \mathrm{CH}_{d}\right), 1.78(2 \mathrm{H}, \mathrm{br} \mathrm{s}$, $\left.\mathrm{C}(\mathrm{O})-\mathrm{CH}_{a} \mathrm{H}_{\mathrm{b}}\right), 1.66\left(1 \mathrm{H}, \mathrm{ddd}, 12.1 \mathrm{~Hz}, 8.7 \mathrm{~Hz}, 3.7 \mathrm{~Hz}, 6-\mathrm{CH}_{\mathrm{c}} \mathrm{CH}_{d}\right), 1.48(1 \mathrm{H}, \mathrm{ddd}, 12.1 \mathrm{~Hz}$, $\left.8.9 \mathrm{~Hz}, 3.8 \mathrm{~Hz}, 6-\mathrm{CH}_{\mathrm{c}} \mathrm{CH}_{\mathrm{d}}\right), 1.17\left(3 \mathrm{H}, \mathrm{s}, 9-\mathrm{CH}_{3}\right), 1.09(1 \mathrm{H}, \mathrm{ddd}, 12.1 \mathrm{~Hz}, 8.8 \mathrm{~Hz}, 3.6 \mathrm{~Hz}$, $\left.5-\mathrm{CH}_{c} \mathrm{CH}_{\mathrm{d}}\right), 0.89\left(3 \mathrm{H}, \mathrm{s}, 10-\mathrm{CH}_{3}\right), 0.86\left(3 \mathrm{H}, \mathrm{s}, 8-\mathrm{CH}_{3}\right) ;{ }^{13} \mathrm{C} \mathrm{NMR}\left(125 \mathrm{MHz}, \mathrm{CDCl}_{3}\right): 207.7$, 166.6/ , 165.3, 143.9, 136.9/136.6, 55.6, 54.5/51.1, 52.6, 50.2/49.1, 31.7, 30.1/29.6, 28.3/28.0, 25.2, 19.5, 19.1, 11.2. MS m/z (rel. int.): 287 (97, [M] $\left.]^{+}\right), 272$ (47), 258 (25), 230 (8), 202 (6), 163 (100), 135 (91), 91 (75), 67 (37). IR (KBr, $\left.v\left(\mathrm{~cm}^{-1}\right)\right)$ : 1721 (CO), 1620 (C=C), 1578 (Amide 
I.), 1427 .

(1R,5S)-8-Benzoyl-8-azabicyclo-[3.2.1]octan-3-one (6a), yield: $93 \mathrm{mg}(81 \%)$; off white solid, m.p. $108-109{ }^{\circ} \mathrm{C}$, [Anal. Calcd. for $\mathrm{C}_{14} \mathrm{H}_{15} \mathrm{NO}_{2}$ : $\mathrm{C}$, 73.34; $\mathrm{H}, 6.59 ; \mathrm{N}, 6.11$. Found: $\mathrm{C}, 72.82$; $\mathrm{H}, 6.45 ; \mathrm{N}, 6.16] ; R_{\mathrm{f}}\left(30 \%\right.$ EtOAc, $\left.70 \% \mathrm{CHCl}_{3}\right) 0.52 ;{ }^{1} \mathrm{H}$ NMR $\left(500 \mathrm{MHz}, \mathrm{CDCl}_{3}\right)$ : 7.56-7.52 ( $2 \mathrm{H}, \mathrm{m}, \mathrm{Ar}-\mathrm{H}), 7.51-7.41(3 \mathrm{H}, \mathrm{m}, \mathrm{Ar}-\mathrm{H}), 5.07(1 \mathrm{H}, \mathrm{br} \mathrm{s}, \mathrm{N}-\mathrm{CH}), 4.41(1 \mathrm{H}, \mathrm{br} \mathrm{s}, \mathrm{N}-\mathrm{CH}), 2.95$ $(1 \mathrm{H}, \mathrm{br} \mathrm{s}, \mathrm{CH}), 2.47\left(2 \mathrm{H}, \mathrm{br} \mathrm{s}, \mathrm{CH}_{2}\right), 2.34(1 \mathrm{H}, \mathrm{br} \mathrm{s}, \mathrm{CH}), 2.17\left(2 \mathrm{H}, \mathrm{br} \mathrm{s}, \mathrm{CH}_{2}\right), 1.77(2 \mathrm{H}, \mathrm{d}$, $\left.8.2 \mathrm{~Hz}, \mathrm{CH}_{2}\right) ;{ }^{13} \mathrm{C} \mathrm{NMR}\left(125 \mathrm{MHz}, \mathrm{CDCl}_{3}\right): 207.5,168.9,135.5,130.6,128.7$ (2 C), $127.1(2 \mathrm{C})$, 56.0, 51.5, 49.7, 48.8, 29.6, 28.1. MS m/z (rel. int.): 229 (14, [M] $\left.]^{+}\right), 200$ (4), 172 (7), 124 (33), 105 (100), 77 (49), 51 (13). IR (KBr, $v\left(\mathrm{~cm}^{-1}\right)$ ): 1719 (CO), 1643 (C=C), 1621 (Amide I.), 1422.

1-((1R,5S)-3-oxo-8-azabicyclo[3.2.1]octan-8-yl)-2-phenylethane-1,2-dione (6a'), yield: $57 \mathrm{mg}$ (44\%); off white viscous material, [Anal. Calcd. for $\mathrm{C}_{15} \mathrm{H}_{15} \mathrm{NO}_{3}$ : C, 70.02; $\mathrm{H}, 5.88 ; \mathrm{N}, 5.44$.]; $R_{\mathrm{f}}\left(20 \%\right.$ EtOAc, $\left.80 \% \mathrm{CHCl}_{3}\right)$ 0.57; ${ }^{1} \mathrm{H}$ NMR $\left(500 \mathrm{MHz}, \mathrm{CDCl}_{3}\right): 8.05(2 \mathrm{H}, \mathrm{dd}, 8.4 \mathrm{~Hz}$, $\left.1.2 \mathrm{~Hz}, \mathrm{H}_{\text {ortho }}(\mathrm{Ph})\right), 7.69\left(1 \mathrm{H}, \mathrm{t}, 7.4 \mathrm{~Hz}, \mathrm{H}_{\text {para }}(\mathrm{Ph})\right), 7.55\left(2 \mathrm{H}, \mathrm{t}, 7.6 \mathrm{~Hz}, \mathrm{H}_{\text {meta }}(\mathrm{Ph})\right), 5.16-5.08$ $(1 \mathrm{H}, \mathrm{m}, \mathrm{N}-\mathrm{CH}), 4.36-4.28(1 \mathrm{H}, \mathrm{m}, \mathrm{N}-\mathrm{CH}), 2.93-2.85(2 \mathrm{H}, \mathrm{m}, 2 \times \mathrm{CH}), 2.54(1 \mathrm{H}, \mathrm{d}, 16.4 \mathrm{~Hz}$, $\mathrm{CH}), 2.42(1 \mathrm{H}, \mathrm{d}, 16.4 \mathrm{~Hz}, \mathrm{CH}), 2.26-2.08(2 \mathrm{H}, \mathrm{br} \mathrm{m}, 2 \times \mathrm{CH}), 1.87-1.79(2 \mathrm{H}, \mathrm{m}, 2 \times \mathrm{CH})$; ${ }^{13} \mathrm{C}$ NMR (125 MHz, $\mathrm{CDCl}_{3}$ ): 206.4, 190.7, 162.3, 135.0, 132.9, 129.9 (2 C), 129.1 (2 C), 54.4, 51.2, 49.9, 49.1, 29.7, 27.9. MS m/z (rel. int.): 257 (8, [M] $\left.{ }^{+}\right), 152$ (13), 124 (1), 105 (100), 77 (38), $67(20), 51(13)$. IR (KBr, $\left.v\left(\mathrm{~cm}^{-1}\right)\right)$ : 1751 and $1718(\mathrm{CO}), 1653(\mathrm{C}=\mathrm{C}), 1628$ (Amide I.), 1452 .

(1R,5S)-8-Picolinoyl-8-azabicyclo-[3.2.1]octan-3-one (7a), yield: $103 \mathrm{mg}$ (90\%); beige solid, m.p. 98-99 ${ }^{\circ} \mathrm{C}$, [Anal. Calcd. for $\mathrm{C}_{13} \mathrm{H}_{14} \mathrm{~N}_{2} \mathrm{O}_{2}: \mathrm{C}, 67.81 ; \mathrm{H}, 6.13 ; \mathrm{N}, 12.17$. Found: $\mathrm{C}, 67.38 ; \mathrm{H}$, 6.13; N, 12.30]; $R_{\mathrm{f}}\left(3 \% \mathrm{MeOH}, 97 \% \mathrm{CHCl}_{3}\right) 0.48 ;{ }^{1} \mathrm{H} \mathrm{NMR}\left(500 \mathrm{MHz}, \mathrm{CDCl}_{3}\right): 8.61(1 \mathrm{H}$, ddd, $4.8 \mathrm{~Hz}, 1.7 \mathrm{~Hz}, 0.9 \mathrm{~Hz}, \mathrm{Py}-\mathrm{H}), 7.95(1 \mathrm{H}, \mathrm{ddd}, 7.8 \mathrm{~Hz}, 1.7 \mathrm{~Hz}, 1.0 \mathrm{~Hz}, \mathrm{Py}-\mathrm{H}), 7.85(1 \mathrm{H}, \mathrm{td}$, $7.8 \mathrm{~Hz}, 1.7 \mathrm{~Hz}, \mathrm{Py}-\mathrm{H}), 7.41(1 \mathrm{H}, \mathrm{ddd}, 7.8 \mathrm{~Hz}, 4.8 \mathrm{~Hz}, 1.2 \mathrm{~Hz}, \mathrm{Py}-\mathrm{H}), 5.24-5.13(2 \mathrm{H}, \mathrm{m}, \mathrm{N}-\mathrm{CH})$, $3.08(1 \mathrm{H}, \mathrm{dd}, 16.1 \mathrm{~Hz}, 4.5 \mathrm{~Hz}, \mathrm{CH}), 2.90(1 \mathrm{H}, \mathrm{dd}, 16.1 \mathrm{~Hz}, 4.4 \mathrm{~Hz}, \mathrm{CH}), 2.46(2 \mathrm{H}, \mathrm{q}, 16.1 \mathrm{~Hz}$, $\left.\mathrm{CH}_{2}\right), 2.24-2.10\left(2 \mathrm{H}, \mathrm{m}, \mathrm{CH}_{2}\right), 1.89-1.73\left(2 \mathrm{H}, \mathrm{m}, \mathrm{CH}_{2}\right) ;{ }^{13} \mathrm{C} \mathrm{NMR}\left(125 \mathrm{MHz}, \mathrm{CDCl}_{3}\right)$ : 208.3, 164.3, 153.5, 148.1, 137.1, 125.3, 124.5, 55.2, 52.2, 50.3, 49.1, 30.0, 27.5. MS m/z (rel. int.): $230\left(37,[\mathrm{M}]^{+}\right), 202(8), 174$ (8), 161 (13), 133 (4), 124 (16), 106 (36), 93 (19), 78 (100), 51 (22). IR (KBr, $\left.v\left(\mathrm{~cm}^{-1}\right)\right)$ : 1718 (CO), 1629 (Amide I.), 1617 (C=C), 1586, 1472, 1448, 1420, 1411.

(1R,5S)-8-Nicotinoyl-8-azabicyclo-[3.2.1]octan-3-one (8a), yield: $94 \mathrm{mg}$ (82\%); yellowish oil, [Anal. Calcd. for $\mathrm{C}_{13} \mathrm{H}_{14} \mathrm{~N}_{2} \mathrm{O}_{2}$ : C, 67.81; $\mathrm{H}, 6.13 ; \mathrm{N}, 12.17$. Found: $\mathrm{C}, 67.52 ; \mathrm{H}, 6.09 ; \mathrm{N}$, 12.30]; $R_{\mathrm{f}}\left(6 \% \mathrm{MeOH}, 94 \% \mathrm{CHCl}_{3}\right)$ 0.45; ${ }^{1} \mathrm{H} \mathrm{NMR}\left(500 \mathrm{MHz}, \mathrm{CDCl}_{3}\right): 8.78(1 \mathrm{H}, \mathrm{dd}, 2.2 \mathrm{~Hz}$, $0.7 \mathrm{~Hz}, \mathrm{Py}-\mathrm{H}), 8.69$ (1 H, dd, 4.9 Hz, $1.8 \mathrm{~Hz}, \mathrm{Py}-\mathrm{H}), 7.87$ (1 H, dt, $7.9 \mathrm{~Hz}, 1.9 \mathrm{~Hz}, \mathrm{Py}-\mathrm{H}), 7.38$ $(1 \mathrm{H}, \mathrm{ddd}, 7.9 \mathrm{~Hz}, 4.9 \mathrm{~Hz}, 0.8 \mathrm{~Hz}, \mathrm{Py}-\mathrm{H}), 5.05(1 \mathrm{H}, \mathrm{br} \mathrm{s}, \mathrm{N}-\mathrm{CH}), 4.36(1 \mathrm{H}, \mathrm{br}$ s, N-CH), 2.90 $\left(1 \mathrm{H}\right.$, br s, CH) $2.64-2.26(3 \mathrm{H}$, br m $3 \times \mathrm{CH}), 2.21-2.09\left(2 \mathrm{H}, \mathrm{m}, \mathrm{CH}_{2}\right), 1.77(2 \mathrm{H}, \mathrm{d}, 8.4 \mathrm{~Hz}$, $\left.\mathrm{CH}_{2}\right) ;{ }^{13} \mathrm{C}$ NMR (125 MHz, $\left.\mathrm{CDCl}_{3}\right): 206.6,166.2,151.6,148.0,135.1,131.3,123.6,56.1,51.6$, 49.6, 48.8, 29.7, 28.0. MS m/z (rel. int.): $230\left(13,[\mathrm{M}]^{+}\right), 201$ (3), 173 (15), 147 (2), 124 (51), 106 (100), 78 (64), 51 (28). IR (KBr, $\left.v\left(\mathrm{~cm}^{-1}\right)\right)$ : 1717 (CO), 1651 (C=C), 1631 (Amide I.), 1589, $1436,1411$.

(1R,5S)-8-(Thiophene-2-carbonyl)-8-azabicyclo[3.2.1]octan-3-one (9a), yield: $96 \mathrm{mg}$ (82\%); beige solid, m.p. $71-72{ }^{\circ} \mathrm{C}$, [Anal. Calcd. for $\mathrm{C}_{12} \mathrm{H}_{13} \mathrm{NO}_{2} \mathrm{~S}$ : C, 61.25; H, 5.57; N, 5.95. Found: $\mathrm{C}$, 61.04; $\mathrm{H}, 5.51 ; \mathrm{N}, 6.09] ; R_{\mathrm{f}}\left(20 \% \mathrm{EtOAc}, 80 \% \mathrm{CHCl}_{3}\right) 0.40 ;{ }^{1} \mathrm{H} \mathrm{NMR}\left(500 \mathrm{MHz}, \mathrm{CDCl}_{3}\right): 7.53-$ $7.49(2 \mathrm{H}, \mathrm{m}$, overlapping of two thiophenyl-H), $7.09(1 \mathrm{H}, \mathrm{dd}, 5.1 \mathrm{~Hz}, 3.9 \mathrm{~Hz}$, thiophenyl-H), $4.99(2 \mathrm{H}, \mathrm{br} \mathrm{s}, \mathrm{N}-\mathrm{CH}), 2.80\left(2 \mathrm{H}, \mathrm{d}, 16.1 \mathrm{~Hz}, \mathrm{CH}_{2}\right), 2.45\left(2 \mathrm{H}, \mathrm{d}, 16.1 \mathrm{~Hz}, \mathrm{CH}_{2}\right), 2.22-2.11(2$ $\mathrm{H}, \mathrm{m} \mathrm{CH} 2), 1.82-1.75\left(2 \mathrm{H}, \mathrm{m}, \mathrm{CH}_{2}\right) ;{ }^{13} \mathrm{C} \mathrm{NMR}\left(125 \mathrm{MHz}, \mathrm{CDCl}_{3}\right): 207.2,161.4,137.2,129.8$, 129.5, 54.0 (2 C), 49.3 (2 C), 28.8 (2 C). MS m/z (rel. int.): 235 (11, [M] $\left.{ }^{+}\right), 207$ (1), 178 (5), 124 (28), 111 (100), 97 (2), 83 (9), 68 (3), 54 (3). IR (KBr, $\left.v\left(\mathrm{~cm}^{-1}\right)\right): 1717$ (CO), 1652 (C=C), 1606 (Amide I.), 1522, 1432, 1406. 
(1R,5S)-8-(1H-Indole-5-carbonyl)-8-azabicyclo[3.2.1]octan-3-one (10a), yield: $91 \mathrm{mg}(68 \%)$; offwhite solid, m.p. $149-150{ }^{\circ} \mathrm{C}$, [Anal. Calcd. for $\mathrm{C}_{16} \mathrm{H}_{16} \mathrm{~N}_{2} \mathrm{O}_{2}$ : C, 71.62; H, 6.01; N, 10.44 . Found: C, 71.45; H, 5.92; N, 10.35]; $R_{\mathrm{f}}(99 \%$ EtOAc, $1 \% \mathrm{MeOH}) 0.59 ;{ }^{1} \mathrm{H}$ NMR (500 MHz, $\left.\mathrm{CDCl}_{3}\right)$ : $9.33(1 \mathrm{H}, \mathrm{br} \mathrm{s}$, indole-NH), $7.89(1 \mathrm{H}, \mathrm{s}, \mathrm{Ar}-\mathrm{H}), 7.37(1 \mathrm{H}, \mathrm{dd}, 8.3 \mathrm{~Hz}, 1.5 \mathrm{~Hz}, \mathrm{Ar}-\mathrm{H})$, $7.33(1 \mathrm{H}, \mathrm{d}, 8.4 \mathrm{~Hz}, \mathrm{Ar}-\mathrm{H}), 7.23(1 \mathrm{H}, \mathrm{dd}, 3.3 \mathrm{~Hz}, 2.6 \mathrm{~Hz}, \mathrm{Ar}-\mathrm{H}), 6.59-6.56$ (1 H, m, Ar-H) $5.04(1 \mathrm{H}$, br s, N-CH), $4.63(1 \mathrm{H}$, br s, N-CH), $2.93(1 \mathrm{H}$, br s, $\mathrm{CH}), 2.67(1 \mathrm{H}$, br s, $\mathrm{CH}), 2.40$ (2 $\mathrm{H}$, br s, $\left.\mathrm{CH}_{2}\right), 2.26-2.14(2 \mathrm{H}, \mathrm{m} \mathrm{CH}), 1.83-1.72\left(2 \mathrm{H}, \mathrm{m}, \mathrm{CH}_{2}\right) ;{ }^{13} \mathrm{C} \mathrm{NMR}\left(125 \mathrm{MHz}_{2} \mathrm{CDCl}_{3}\right)$ : 208.1, 170.9, 137.1, 127.4, 126.7, 126.0, 121.1, 120.4, 111.4, 103.0, 56.2, 51.7, 49.3 (2 C), 28.7 (2 C). MS m/z (rel. int.): 268 (15, [M] $\left.]^{+}\right), 239$ (1), 144 (100), 124 (4), 116 (27), 89 (10), 62 (3). IR $\left(\mathrm{KBr}, v\left(\mathrm{~cm}^{-1}\right)\right)$ : $3318(\mathrm{NH}), 1706$ (CO), 1629 (C=C), 1594 (Amide I.), 1431.

Cyclohex-1-en-1-yl((1R,3R,5S)-3-hydroxy-8-azabicyclo[3.2.1]octan-8-yl)methanone (1b), yield: $105 \mathrm{mg}$ (89\%); beige solid, m.p. $86-87^{\circ} \mathrm{C}$, [Anal. Calcd. for $\mathrm{C}_{14} \mathrm{H}_{21} \mathrm{NO}_{2}$ : C, 71.46; $\mathrm{H}, 9.00$; $\mathrm{N}, 5.95$. Found: C, 71.31; $\mathrm{H}, 6.03 ; \mathrm{N}, 9.18] ; R_{\mathrm{f}}\left(8 \% \mathrm{MeOH}, 92 \% \mathrm{CHCl}_{3}\right) 0.38 ;{ }^{1} \mathrm{H} \mathrm{NMR}(500$ $\left.\mathrm{MHz}_{\mathrm{CDCl}}\right)$ : $5.84-5.77(1 \mathrm{H}, \mathrm{m}, \mathrm{C}=\mathrm{CH}), 4.58(1 \mathrm{H}, \mathrm{br} \mathrm{s}, \mathrm{N}-\mathrm{CH}), 4.16(1 \mathrm{H}$, br s, N-CH $), 4.08$ $(1 \mathrm{H}, \mathrm{t}, 4.8 \mathrm{~Hz}, \mathrm{CH}-\mathrm{OH}), 3.09(1 \mathrm{H}, \mathrm{br} \mathrm{s}, \mathrm{OH}), 2.29-1.54(16 \mathrm{H}$, skeleton protons of nortropine and cyclohexen-1-yl ring), ${ }^{13} \mathrm{C} \mathrm{NMR}\left(125 \mathrm{MHz} \mathrm{CDCl}_{3}\right): 169.2,134.9,127.6,64.6,55.5,50.4$, 40.5, 38.7, 28.6, 27.1, 25.9, 24.6, 22.1, 21.6. MS m/z (rel. int.): 235 (39, [M] $\left.{ }^{+}\right), 218$ (5), 207 (15), 154 (7), 126 (19), 109 (100), 81 (77), 68 (31), 53 (16). IR (KBr, v (cm $\left.\left.{ }^{-1}\right)\right)$ : $3426(\mathrm{OH}), 1655$ $(\mathrm{C}=\mathrm{C}), 1592$ (Amide I.), 1438, 1087 (C-O), $1046 \gamma(\mathrm{OH})$.

(4-(tert-butyl)cyclohex-1-en-1-yl)((1R,3R,5S)-3-hydroxy-8-azabicyclo[3.2.1]octan-8-yl)methanone (2b), yield: $115 \mathrm{mg}$ (79\%); off-white solid, m.p. 133-134 ${ }^{\circ} \mathrm{C}$, [Anal. Calcd. for $\mathrm{C}_{18} \mathrm{H}_{29} \mathrm{NO}_{2}$ : $\mathrm{C}, 74.18 ; \mathrm{H}, 10.03 ; \mathrm{N}, 4.81$. Found: $\mathrm{C}, 73.31 ; \mathrm{H}, 10.01 ; \mathrm{N}, 5.16] ; \mathrm{R}_{\mathrm{f}}\left(5 \% \mathrm{MeOH}, 95 \% \mathrm{CHCl}_{3}\right)$ $0.45 ;{ }^{1} \mathrm{H}$ NMR $\left(500 \mathrm{MHz} \mathrm{CDCl}_{3}\right): 5.85(1 \mathrm{H}$, br s, $\mathrm{C}=\mathrm{CH}), 4.68(1 \mathrm{H}, \mathrm{br} \mathrm{s}, \mathrm{N}-\mathrm{CH}), 4.23(1 \mathrm{H}, \mathrm{br}$ s, N-CH), 4.18 (1 H, br s, CH-OH), 2.89 (1 H, br s, OH), 2.43-1.71 (14 H, skeleton protons of nortropine and cyclohexen-1-yl ring), 1.34-1.24 (1 H, m, Cy-CH), 1.23-1.13 (1 H, m, Cy-CH), $0.87\left(9 \mathrm{H}, \mathrm{C}\left(\mathrm{CH}_{3}\right)_{3}\right),{ }^{13} \mathrm{C} \mathrm{NMR}\left(125 \mathrm{MHz} \mathrm{CDCl}_{3}\right): 169.1,134.7,128.1,64.7,55.5,50.3,43.5$, 40.5, 38.7, 32.2, 28.7, 27.7, 27.4, 27.1, 26.5, 23.6. MS m/z (rel. int.): $291\left(80,[\mathrm{M}]^{+}\right), 278(24)$, 263 (13), 234 (51), 206 (16), 165 (77), 137 (6), 126 (38), 110 (97), 81 (65), 68 (57), 57 (100). IR $\left(\mathrm{KBr}, v\left(\mathrm{~cm}^{-1}\right)\right): 3427(\mathrm{OH}), 1661$ (C=C), 1584 (Amide I.), 1465, $1093(\mathrm{C}-\mathrm{O}), 1047 \gamma(\mathrm{OH})$.

(E)-1-((1R,3R,5S)-3-hydroxy-8-azabicyclo[3.2.1]octan-8-yl)non-2-en-1-one (3b), yield: $86 \mathrm{mg}$ (65\%); yellowish oil, [Anal. Calcd. for $\mathrm{C}_{16} \mathrm{H}_{27} \mathrm{NO}_{2}: \mathrm{C}, 72.41 ; \mathrm{H}, 10.25 ; \mathrm{N}, 5.28$. Found: $\mathrm{C}$, 71.58; $\mathrm{H}, 10.33 ; \mathrm{N}, 5.33] ; R_{\mathrm{f}}\left(5 \% \mathrm{MeOH}, 95 \% \mathrm{CHCl}_{3}\right) 0.51 ;{ }^{1} \mathrm{H} \mathrm{NMR}\left(500 \mathrm{MHz}, \mathrm{CDCl}_{3}\right)$ : $6.91(1 \mathrm{H}, \mathrm{dt}, J 15.1 \mathrm{~Hz}, 7.1 \mathrm{~Hz},=\mathrm{CH}), 6.13(1 \mathrm{H}, \mathrm{dt}, J 15.1 \mathrm{~Hz}, 1.4 \mathrm{~Hz},=C H), 4.67(1 \mathrm{H}, \mathrm{br} \mathrm{s}$, $\mathrm{N}-\mathrm{CH}), 4.29$ (1 H, br s, N-CH), $4.12(1 \mathrm{H}, \mathrm{t}, 4.8 \mathrm{~Hz}, \mathrm{CH}-\mathrm{OH}), 2.52(1 \mathrm{H}, \mathrm{br} \mathrm{s}, \mathrm{OH}), 2.40-1.74$ $\left(10 \mathrm{H}\right.$, protons of nortropine skeleton and $\mathrm{CH}_{2}$ of octene chain), $1.50-1.39\left(2 \mathrm{H}, \mathrm{m}, \mathrm{CH}_{2}\right)$, 1.37-1.22 (6 H, br m, $\left.3 \times \mathrm{CH}_{2}\right), 0.89\left(3 \mathrm{H}, \mathrm{t}, 6.9 \mathrm{~Hz}, \mathrm{CH}_{2} \mathrm{CH}_{3}\right) ;{ }^{13} \mathrm{C} \mathrm{NMR}\left(125 \mathrm{MHz}, \mathrm{CDCl}_{3}\right)$ : $162.7,146.3,120.6,64.9,53.9,51.1,40.5,38.6,32.4,31.6,28.9,28.8,28.4,27.0,22.6,14.1$. MS m/z (rel. int.): 265 (17, [M] $\left.]^{+}\right), 236$ (5), 208 (9), 194 (9), 180 (25), 165 (11), 139 (26), 126 (39), 110 (47), 82 (41), 68 (77), 55 (100). IR (KBr, $v\left(\mathrm{~cm}^{-1}\right)$ ): $3409(\mathrm{OH}), 1658(\mathrm{C}=\mathrm{C}), 1596$ (Amide I.), 1448, $1091(\mathrm{C}-\mathrm{O}), 1043 \gamma(\mathrm{OH})$.

(((10.S,13S)-10,13-dimethyl-2,3,4,5,6,7,8,9,10,11,12,13,14,15-tetradecahydro-1H-cyclopenta[a]phe nanthren-17-yl)((1R,3S,5S)-3-hydroxy-8-azabicyclo[3.2.1]octan-8-yl)methanone (4b) (1:1 ratio of diastereomers), yield: $113 \mathrm{mg}(55 \%)$; white solid, m.p. $>260{ }^{\circ} \mathrm{C}$ [Anal. Calcd. for $\mathrm{C}_{27} \mathrm{H}_{41} \mathrm{NO}_{2}$ : C, 78.78; H, 10.04; N, 3.40. Found: C, 78.26; H, 9.98; N, 3.50]; $\mathrm{R}_{\mathrm{f}}(2 \% \mathrm{MeOH}, 98$ \% $\mathrm{CHCl}_{3}$ ) 0.58; ${ }^{1} \mathrm{H}$ NMR (500 MHz, $\left.\mathrm{CDCl}_{3}\right): 5.84(1 \mathrm{H}$, br s, $=\mathrm{CH}), 4.79 / 4.68(1 \mathrm{H}$, br s, N$\mathrm{CH}), 4.30 / 4.24$ (1 H, br s, N-CH), 4.19 (1 H, br s, CH-OH), 2.33-0.69 (37 H, skeleton protons of steroid and nortropine $+\mathrm{OH}) ;{ }^{13} \mathrm{C} \mathrm{NMR}\left(125 \mathrm{MHz}, \mathrm{CDCl}_{3}\right): 165.3 / 165.0,150.2 / 149.6$, 130.5, 65.4, 56.9, 55.7/55.0, 55.4, 50.2/49.8, 48.5/48.4, 47.4, 40.8/40.5, 39.3/38.8, 38.6, 36.5, $34.9 / 34.3,33.9,32.2,32.0 / 28.6,29.1,29.0,27.5 / 27.0,26.8,22.2,20.6,16.8,12.2 . \mathrm{MS} \mathrm{m} / \mathrm{z}$ (rel. int.): $411\left(100,[\mathrm{M}]^{+}\right), 396$ (82), 368 (4), 326 (2), 285 (20), 246 (4), 207 (12), 161 (5), 126 (10), 93 
(12), 68 (10), 55 (8). IR (KBr, $\left.v\left(\mathrm{~cm}^{-1}\right)\right)$ : $3355(\mathrm{OH}), 1613$ (C=C), 1575 (Amide I.), 1435, 1096 (C-O), $1046 \gamma(\mathrm{OH})$.

$((1 R, 3 S, 5 S)$-3-hydroxy-8-azabicyclo[3.2.1]octan-8-yl)((1S,4R)-1,7,7-trimethylbicyclo[2.2.1]hept-2en-2-yl)methanone $(\mathbf{5 b})$ (1:1 ratio of diastereomers), yield: $91 \mathrm{mg}(63 \%)$; white solid, m.p. 135-136 ${ }^{\circ} \mathrm{C}$ [Anal. Calcd. for $\mathrm{C}_{18} \mathrm{H}_{27} \mathrm{NO}_{2}$ : C, 74.70; $\mathrm{H}, 9.40 ; \mathrm{N}, 4.84$. Found: $\mathrm{C}, 74.26 ; \mathrm{H}$, 9.36; N, 4.83]; $R_{\mathrm{f}}\left(5 \% \mathrm{MeOH}, 95 \% \mathrm{CHCl}_{3}\right) 0.48 ;{ }^{1} \mathrm{H} \mathrm{NMR}\left(500 \mathrm{MHz}, \mathrm{CDCl}_{3}\right): 6.01(1 \mathrm{H}, \mathrm{s}$, $=\mathrm{CH}), 4.79 / 4.60(1 \mathrm{H}, \mathrm{br} \mathrm{s}, \mathrm{N}-\mathrm{CH}), 4.42 / 4.39(1 \mathrm{H}, \mathrm{brs}, \mathrm{N}-\mathrm{CH}), 4.18(1 \mathrm{H}, \mathrm{s}, \mathrm{CH}-\mathrm{OH}), 2.44$ $(1 \mathrm{H}, \mathrm{s}, \mathrm{OH}), 2.33-1.75(10 \mathrm{H}, \mathrm{br} \mathrm{m}, \mathrm{CH}$ protons of nortropine and bornene skeleton), 1.62 $\left(1 \mathrm{H}, \mathrm{ddd}, 12.1 \mathrm{~Hz}, 8.5 \mathrm{~Hz}, 3.7 \mathrm{~Hz}, 6-\mathrm{CH}_{\mathrm{c}} \mathrm{CH}_{d}\right), 1.46(1 \mathrm{H}$, ddd, $12.1 \mathrm{~Hz}, 8.5 \mathrm{~Hz}, 3.7 \mathrm{~Hz}$, 6- $\left.\mathrm{CH}_{\mathrm{c}} \mathrm{CH}_{\mathrm{d}}\right), 1.13\left(3 \mathrm{H}, \mathrm{s}, 9-\mathrm{CH}_{3}\right), 1.06\left(1 \mathrm{H}, \mathrm{ddd}, 12.1 \mathrm{~Hz}, 8.5 \mathrm{~Hz}, 3.7 \mathrm{~Hz}, 5-\mathrm{CH}_{c} \mathrm{CH}_{\mathrm{d}}\right), 0.87$ $\left(3 \mathrm{H}, \mathrm{s}, 10-\mathrm{CH}_{3}\right), 0.82\left(3 \mathrm{H}, \mathrm{s}, 8-\mathrm{CH}_{3}\right) ;{ }^{13} \mathrm{C} \mathrm{NMR}\left(125 \mathrm{MHz}, \mathrm{CDCl}_{3}\right): 165.4 / 164.5,144.3 / 144.2$, 135.2/135.1, 65.2, 55.7, 55.6/55.5, 54.4, 52.5, 50.5/49.8, 40.9/40.7, 39.2/38.6, 31.7, 29.1/28.7, 27.7/26.7, 25.3, 19.6/19.4, 19.2, 11.2. MS m/z (rel. int.): 289 (100, [M] $\left.{ }^{+}\right), 274$ (62), 260 (28), 246 (35), 220 (3), 188 (1), 163 (77), 135 (92), 119 (32), 91 (64), 67 (27), 54 (27). IR (KBr, v $\left.\left(\mathrm{cm}^{-1}\right)\right): 3425(\mathrm{OH}), 1617(\mathrm{C}=\mathrm{C}), 1576$ (Amide I.), 1443, $1088(\mathrm{C}-\mathrm{O}), 1046 \gamma(\mathrm{OH})$.

((1R,3R,5S)-3-hydroxy-8-azabicyclo[3.2.1]octan-8-yl)(phenyl)methanone (6b), yield: $78 \mathrm{mg}(68 \%)$; off-white solid, m.p. $126-127^{\circ} \mathrm{C}$, [Anal. Calcd. for $\mathrm{C}_{14} \mathrm{H}_{17} \mathrm{NO}_{2}$ : C, 72.70; $\mathrm{H}, 7.41 ; \mathrm{N}, 6.06$. Found: C, 71.90; H, 7.27; N, 6.17]; $R_{\mathrm{f}}\left(2 \% \mathrm{MeOH}, 98 \% \mathrm{CHCl}_{3}\right) 0.51 ;{ }^{1} \mathrm{H} \mathrm{NMR}(500 \mathrm{MHz}$, $\left.\mathrm{CDCl}_{3}\right)$ : 7.50-7.37 (5 H, m, Ar-H), $4.80(1 \mathrm{H}, \mathrm{br} \mathrm{s}, \mathrm{N}-\mathrm{CH}), 4.18(1 \mathrm{H}, \mathrm{br} \mathrm{s}, \mathrm{CH}-\mathrm{OH}), 4.06(1 \mathrm{H}$, br s, N-CH), 2.37-1.71 (9 H, protons of nortropine skeleton); ${ }^{13} \mathrm{C} \mathrm{NMR}\left(125 \mathrm{MHz}, \mathrm{CDCl}_{3}\right)$ : 167.9, 136.5, 129.9, 128.4 (2 C), 127.0 (2 C), 64.9, 56.0, 51.0, 40.3, 38.7, 28.6, 27.3. MS m/z (rel. int.): $231\left(20,[\mathrm{M}]^{+}\right), 207$ (1), 186 (4), 154 (1), 127 (12), 105 (100), 77 (56), 68 (18), 51 (13). IR $\left(\mathrm{KBr}, v\left(\mathrm{~cm}^{-1}\right)\right): 3337(\mathrm{OH}), 1607(\mathrm{C}=\mathrm{C}), 1596$ (Amide I.), 1448, $1084(\mathrm{C}-\mathrm{O}), 1041 \gamma(\mathrm{OH})$.

1-((1R,3R,5S)-3-hydroxy-8-azabicyclo[3.2.1]octan-8-yl)-2-phenylethane-1,2-dione (6b'), yield: 39 mg (30\%); beige solid, m.p. $92-93{ }^{\circ} \mathrm{C}$, [Anal. Calcd. for $\mathrm{C}_{15} \mathrm{H}_{17} \mathrm{NO}_{3}$ : C, 69.48; H, 6.61; N, 5.40. Found: $\mathrm{C}, 69.01 ; \mathrm{H}, 6.50 ; \mathrm{N}, 5.47] ; R_{\mathrm{f}}\left(2 \% \mathrm{MeOH}, 98 \% \mathrm{CHCl}_{3}\right) 0.34 ;{ }^{1} \mathrm{H} \mathrm{NMR}(500$ $\left.\mathrm{MHz}, \mathrm{CDCl}_{3}\right): 7.97\left(2 \mathrm{H}, \mathrm{dd}, 8.5 \mathrm{~Hz}, 1.2 \mathrm{~Hz}, \mathrm{H}_{\text {ortho }}(\mathrm{Ph})\right), 7.65\left(1 \mathrm{H}, \mathrm{t}, 7.5 \mathrm{~Hz}, \mathrm{H}_{\text {para }}(\mathrm{Ph})\right), 7.51$ $\left(2 \mathrm{H}, \mathrm{t}, 7.5 \mathrm{~Hz}, \mathrm{H}_{\text {meta }}(\mathrm{Ph})\right), 4.81-4.76(1 \mathrm{H}, \mathrm{m}, \mathrm{N}-\mathrm{CH}), 4.16(1 \mathrm{H}, \mathrm{t}, 4.8 \mathrm{~Hz}, \mathrm{CH}-\mathrm{OH}), 3.97-3.92$ $(1 \mathrm{H}, \mathrm{m}, \mathrm{N}-\mathrm{CH}), 2.40-1.75$ (9 H, skeleton protons of nortropinone); ${ }^{13} \mathrm{C} \mathrm{NMR}(125 \mathrm{MHz}$, $\left.\mathrm{CDCl}_{3}\right):$ 191.4, 161.7, 134.7, 133.1, 129.7 (2 C), 129.0 (2 C), 64.7, 54.6, 50.9, 40.3, 38.9, 28,5, 27.1. MS m/z (rel. int.): 259 (4, [M] $\left.{ }^{+}\right), 154$ (85), 136 (11), 126 (1), 105 (63), 93 (100), 78 (63), 67 (38), 51 (20). IR (KBr, $\left.\vee\left(\mathrm{cm}^{-1}\right)\right)$ : $3424(\mathrm{OH}), 1681(\mathrm{CO}), 1620$ (Amide I.), 1595 (C=C), 1451, $1082(\mathrm{C}-\mathrm{O}), 1044 \gamma(\mathrm{OH}), 716,662$.

((1R,3R,5S)-3-hydroxy-8-azabicyclo[3.2.1]octan-8-yl)(pyridin-2-yl)methanone (7b), yield: $85 \mathrm{mg}$ (73\%); beige solid, m.p. $164-165{ }^{\circ} \mathrm{C}$, [Anal. Calcd. for $\mathrm{C}_{13} \mathrm{H}_{16} \mathrm{~N}_{2} \mathrm{O}_{2}: \mathrm{C}, 67.22 ; \mathrm{H}, 6.94 ; \mathrm{N}$, 12.06. Found: $\mathrm{C}, 66.78 ; \mathrm{H}, 7.14 ; \mathrm{N}, 12.16] ; R_{\mathrm{f}}\left(5 \% \mathrm{MeOH}, 95 \% \mathrm{CHCl}_{3}\right) 0.41 ;{ }^{1} \mathrm{H}$ NMR (500 $\left.\mathrm{MHz}, \mathrm{CDCl}_{3}\right): 8.62(1 \mathrm{H}, \mathrm{d}, 4.8 \mathrm{~Hz}, \mathrm{Py}-\mathrm{H}), 7.86-7.79(2 \mathrm{H}$, m, overlapping of two Py-H), 7.39 $(1 \mathrm{H}, \mathrm{ddd}, 7.1 \mathrm{~Hz}, 4.8 \mathrm{~Hz}, 1.8 \mathrm{~Hz}, \mathrm{Py}-\mathrm{H}), 4.90(1 \mathrm{H}, \mathrm{brs}, \mathrm{N}-\mathrm{CH}), 4.65(1 \mathrm{H}, \mathrm{brs}, \mathrm{N}-\mathrm{CH}), 4.25$ (1 $\mathrm{H}, \mathrm{t}, 4.8 \mathrm{~Hz}, \mathrm{CH}-\mathrm{OH}), 2.42-2.27\left(4 \mathrm{H}, \mathrm{br} \mathrm{m}, \mathrm{CH}_{2}\right), 2.11-2.00\left(2 \mathrm{H}, \mathrm{br} \mathrm{m}, \mathrm{CH}_{2}\right), 1.91(1 \mathrm{H}, \mathrm{d}$, $14.6 \mathrm{~Hz}, \mathrm{CH}), 1.87-1.73$ (overlapping, $1 \mathrm{H}, \mathrm{d}, 14.6 \mathrm{~Hz}, \mathrm{CH}$ and $1 \mathrm{H}$ of $\mathrm{OH}) ;{ }^{13} \mathrm{C} \mathrm{NMR} \mathrm{(125}$ $\left.\mathrm{MHz}, \mathrm{CDCl}_{3}\right): 163.9,154.3,148.2,136.9,124.7,123.7,64.9,55.3,51.7,40.5,38.9,28.7,26.7$. MS m/z (rel. int.): $232\left(46,[\mathrm{M}]^{+}\right), 216$ (13), 187 (2), 160 (17), 126 (63), 106 (36), 78 (100), 66 (18), 51 (20). IR (KBr, $v\left(\mathrm{~cm}^{-1}\right)$ ): $3354(\mathrm{OH}), 1599$ (Amide I.), 1579 (C=C), 1536, 1479, 1447, 1410, 1089 (C-O), $1038 \gamma(\mathrm{OH})$.

((1R,3R,5S)-3-hydroxy-8-azabicyclo[3.2.1]octan-8-yl)(pyridin-3-yl)methanone (8b), yield: $72 \mathrm{mg}$ (62\%); yellowish solid, m.p. $113-114{ }^{\circ} \mathrm{C}$, [Anal. Calcd. for $\mathrm{C}_{13} \mathrm{H}_{16} \mathrm{~N}_{2} \mathrm{O}_{2}$ : C, 67.22; $\mathrm{H}, 6.94$; $\mathrm{N}, 12.06$. Found: $\mathrm{C}, 66.83 ; \mathrm{H}, 6.69 ; \mathrm{N}, 12.13] ; R_{\mathrm{f}}\left(5 \% \mathrm{MeOH}, 95 \% \mathrm{CHCl}_{3}\right) 0.32 ;{ }^{1} \mathrm{H} \mathrm{NMR}$ (500 MHz, CDCl 3 ): $8.71(1 \mathrm{H}, \mathrm{d}, 1.4 \mathrm{~Hz}, \mathrm{Py}-\mathrm{H}), 8.67(1 \mathrm{H}, \mathrm{dd}, 5.0 \mathrm{~Hz}, 1.4 \mathrm{~Hz}, \mathrm{Py}-\mathrm{H}), 7.82$ $(1 \mathrm{H}, \mathrm{dt}, 7.8 \mathrm{~Hz}, 2.0 \mathrm{~Hz}, \mathrm{Py}-\mathrm{H}), 7.38(1 \mathrm{H}, \mathrm{ddd}, 7.8 \mathrm{~Hz}, 4.8 \mathrm{~Hz} .0 .8 \mathrm{~Hz}, \mathrm{Py}-\mathrm{H}), 4.81$ (1 H, br 
s, N-CH), $4.21(1 \mathrm{H}, \mathrm{t}, 4.8 \mathrm{~Hz}, \mathrm{CH}-\mathrm{OH}), 4.03(1 \mathrm{H}$, br s, N-CH), 2.45-1.77 (9 H, protons of nortropine skeleton and $\mathrm{OH}) ;{ }^{13} \mathrm{C} \mathrm{NMR}\left(125 \mathrm{MHz}, \mathrm{CDCl}_{3}\right)$ : 165.0, 150.8, 147.8, 135.0, 132.2, 123.5, 64.7, 56.3, 51.3, 40.5, 38.7, 28.7, 27.2. MS m/z (rel. int.): 232 (24, [M] $\left.{ }^{+}\right), 213$ (1), 187 (6), 154 (2), $126(18), 106$ (100), 78 (64), 66 (22), 51 (27). IR (KBr, v (cm $\left.\left.{ }^{-1}\right)\right): 3422(\mathrm{OH}), 1612$ (Amide I.), 1591 (C=C), $1443(\mathrm{C}-\mathrm{N}), 1412,1088(\mathrm{C}-\mathrm{O}), 1046 \gamma(\mathrm{OH})$.

$((1 R, 3 R, 5 S)-3-h y d r o x y-8$-azabicyclo[3.2.1]octan-8-yl)(thiophen-2-yl)methanone (9b), yield: 72 mg (92\%); brownish oil, [Anal. Calcd. for $\mathrm{C}_{12} \mathrm{H}_{15} \mathrm{NO}_{2} \mathrm{~S}: \mathrm{C}, 60.73 ; \mathrm{H}, 6.37 ; \mathrm{N}, 5.90$. Found: $\mathrm{C}$, 59.98; H, 6.33; N, 5.98]; $R_{\mathrm{f}}\left(5 \% \mathrm{MeOH}, 95 \% \mathrm{CHCl}_{3}\right)$ 0.49; ${ }^{1} \mathrm{H}$ NMR $\left(500 \mathrm{MHz}, \mathrm{CDCl}_{3}\right): 7.47$ $(1 \mathrm{H}, \mathrm{dd}, 5.0 \mathrm{~Hz}, 1.1 \mathrm{~Hz}, \mathrm{Ar}-\mathrm{H}), 7.44(1 \mathrm{H}, \mathrm{dd}, 3.7 \mathrm{~Hz}, 1.1 \mathrm{~Hz}, \mathrm{Ar}-\mathrm{H}), 7.08(1 \mathrm{H}, \mathrm{dd}, 5.3 \mathrm{~Hz}$, 3.7 Hz, Ar-H), 5.00-4.51 (2 H, overlapping of two br s, N-CH), $4.24(1 \mathrm{H}, \mathrm{t}, 4.8 \mathrm{~Hz}, \mathrm{CH}-\mathrm{OH})$, 2.37-2.28 (2 H, m, CH $), 2.25\left(1 \mathrm{H}\right.$, br s, OH), 2.06-1.99 $\left(2 \mathrm{H}, \mathrm{m}, \mathrm{CH}_{2}\right), 1.91(2 \mathrm{H}, \mathrm{d}, 14.7 \mathrm{~Hz}$, $\left.\mathrm{CH}_{2}\right), 1.69$ (2 H, s, $\left.\mathrm{CH}_{2}\right) ;{ }^{13} \mathrm{C}$ NMR (125 MHz, $\left.\mathrm{CDCl}_{3}\right): 160.2,138.0,129.0,128.7,126.9,64.7$, 55.9, 52.0, 40.2, 36.9, 28.8, 26.7. MS m/z (rel. int.): 237 (17, [M] $\left.]^{+}\right), 218$ (1), 192 (1), 154 (1), 126 (10), 111 (100), 83 (10), 66 (15), 51 (1). IR (KBr, v (cm $\left.\left.{ }^{-1}\right)\right): 3424(\mathrm{OH}), 1587$ (Amide I.), 1522 $(\mathrm{C}=\mathrm{C}), 1443,1087(\mathrm{C}-\mathrm{O}), 1045 \gamma(\mathrm{OH})$.

$((1 R, 3 R, 5 S)-3-h y d r o x y-8$-azabicyclo[3.2.1]octan-8-yl)(1H-indol-5-yl)methanone (10b), yield: 69 mg (51\%); white solid, m. p: 92-93 ${ }^{\circ} \mathrm{C}$, [Anal. Calcd. for $\mathrm{C}_{16} \mathrm{H}_{18} \mathrm{~N}_{2} \mathrm{O}_{2}$ : C, 71.09; $\mathrm{H}, 6.71$; $\mathrm{N}, 10.36$. Found: $\mathrm{C}, 70.95 ; \mathrm{H}, 6.58 ; \mathrm{N}, 10.19] ; R_{\mathrm{f}}\left(5 \% \mathrm{MeOH}, 95 \% \mathrm{CHCl}_{3}\right) 0.49 ;{ }^{1} \mathrm{H} \mathrm{NMR}$ (500 MHz, DMSO-d $\left.d_{6}\right): 11.29$ (1 H, br s, NH), 7.67 (1 H, s, Ar-H), 7.44-7.39 (2 H, m, Ar-H), $7.19(1 \mathrm{H}, \mathrm{dd}, 8.4 \mathrm{~Hz}$ and $1.4 \mathrm{~Hz}, \mathrm{Ar}-\mathrm{H}), 6.50(1 \mathrm{H}, \mathrm{s}, \mathrm{Ar}-\mathrm{H}), 4.62(1 \mathrm{H}, \mathrm{d}, 2.4 \mathrm{~Hz}, \mathrm{CH}-\mathrm{OH})$, 4.55 (1 H, br s, N-CH), $4.10(1 \mathrm{H}$, br s, N-CH), 2.25 (1 H, br s, OH), 2.30-1.55 (8 H, protons of nortropine skeleton); ${ }^{13} \mathrm{C}$ NMR (125 MHz, DMSO-d $\left.d_{6}\right): 168.9,137.0,127.7,127.4,126.9,121.0$, $120.0,111.5,102.3,63.6,56.5,51.2,39.2$ (overlaping with DMSO- $d_{6}$ ), 38.4, 28.7, 27.5. MS m/z (rel. int.): $270\left(19,[\mathrm{M}]^{+}\right), 207$ (1), 160 (11), 144 (100), 116 (25), 89 (10), 62 (2). IR (KBr, v $\left.\left(\mathrm{cm}^{-1}\right)\right)$ : $3414(\mathrm{OH}), 3257(\mathrm{NH}), 1590$ (Amide I.), $1571(\mathrm{C}=\mathrm{C})$, 1440, 1324, $1086(\mathrm{C}-\mathrm{O}), 1045$ $\gamma(\mathrm{OH})$.

\section{Conclusions}

In summary, nortropane-based nucleophiles (nortropinone (a), nortropine (b)) can be used as $N$-nucleophiles in palladium-catalyzed aminocarbonylation. In the presence of simple iodoalkenes, as well as biologically important skeletons possessing iodoalkene functionality, the target carboxamide derivatives have been produced exclusively by using $\mathrm{Pd}(\mathrm{OAc})_{2} / \mathrm{PPh}_{3}$ catalysts. The iodobenzene has shown lower reactivity than the iodoalkene substrates using the above-mentioned catalysts. Thus, increasing the carbon monoxide pressure (40 bar), the chemoselectivity has been shifted toward the corresponding 2-ketocarboxamide formed due to the double $\mathrm{CO}$ insertion, but the conversion was still not complete after $48 \mathrm{~h}$ reaction time. Changing the triphenylphosphine to the bidentate Xantphos, the target carboxamide has been formed selectively under mild reaction conditions ( 1 bar of $\mathrm{CO}, 50-70^{\circ} \mathrm{C}$ ). Using these optimized reaction conditions, we have been able to synthesize various $N$-acylnortropane derivatives in the presence of iodo(hetero)arenes in aminocarbonylation reactions. The new carboxamide derivatives were isolated in moderate to good yields (51-92\%), and they were fully characterized.

It can be stated that the palladium-catalyzed aminocarbonylation provides an efficient tool for the "acylation" of amines possessing biologically important skeletons. It is based on the good acylating ability of the palladium(II)-acyl species formed during the catalytic cycle of the aminocarbonylation. In this way, important carboxamides could be synthesized that cannot be produced by using conventional organic synthetic methods.

Supplementary Materials: ${ }^{1} \mathrm{H}$ and ${ }^{13} \mathrm{C}$ NMR spectra of the products synthesized in this work are available online.

Author Contributions: Conceptualization, L.K. and A.T.; Data curation, Á.E. and A.T.; Formal analysis, L.K. and A.T.; Founding acquisition, L.K. and A.T.; Investigation, L.K., Á.E., H.R. and A.T.; 
Methodology, Á.E., H.R. and A.T.; Resources, L.K.; Writing-original draft A.T.; Writing-review and editing, L.K. and A.T. All authors have read and agreed to the published version of the manuscript.

Funding: This research was financed by the National Research, Development, and Innovation Office (Grant Number PD-132403) and the GINOP-2.3.2-15-2016-00049 grant.

Institutional Review Board Statement: Not applicable.

Informed Consent Statement: Not applicable.

Data Availability Statement: The data presented in this study are available in the Supplementary Materials.

Acknowledgments: The authors thank to G. Mikle for the iodoalkene substrates and V. Takács-Kollár for the graphs. The project has been supported by the European Union, co-financed by the European Social Fund Grant no.: EFOP-3.6.1.-16-2016-00004 entitled by Comprehensive Development for Implementing Smart Specialization Strategies at the University of Pécs. Project no. TKP2020-IKA-08 has been implemented with the support provided from the National Research, Development and Innovation Fund of Hungary, financed under the 2020-4.1.1-TKP2020 funding scheme.

Conflicts of Interest: The authors declare no conflict of interest.

Sample Availability: Samples of the compounds are not available from the authors.

\section{References}

1. Pattabiraman, V.; Bode, J. Rethinking amide bond synthesis. Nature 2011, 480, 471-479. [CrossRef]

2. Kaspar, A.; Reichert, J. Future directions for peptide therapeutics development. Drug Discov. Today 2013, 18, 807-817. [CrossRef] [PubMed]

3. Ghose, A.; Viswanadhan, V.; Wendoloski, J. A knowledge-based approach in designing combinatorial or medicinal chemistry libraries for drug discovery. 1. A qualitative and quantitative characterization of known drug databases. J. Comb. Chem. 1999, 1, 55-68. [CrossRef]

4. De Figueiredo, R.; Suppo, J.; Campagne, J. Nonclassical Routes for Amide Bond Formation. Chem. Rev. 2016, 116, 12029-12122. [CrossRef] [PubMed]

5. Massolo, E.; Pirola, M.; Benaglia, M. Amide Bond Formation Strategies: Latest Advances on a Dateless Transformation. Eur. J. Org. Chem. 2020, 2020, 4641-4651. [CrossRef]

6. Montalbetti, C.; Falque, V. Amide bond formation and peptide coupling. Tetrahedron 2005, 61, 10827-10852. [CrossRef]

7. Challis, B.G.; Challis, J.A. PATAI'S Chemistry of Functional Groups. In Reactions of the Carboxamide Group; Zabicky, J., Ed.; John Wiley \& Sons Ltd.: Hoboken, NJ, USA, 1970.

8. Klausner, Y.; Bodansky, M. The Azide Method in Peptide Synthesis: Its Scope and Limitations. Synthesis 1974, 549-559. [CrossRef]

9. Mikolajczyk, M.; Kielbasinski, P. Recent Developments in the Carbodiimide Chemistry. Tetrahedron 1981, 37, 233-284. [CrossRef]

10. Wittenberger, S.; McLaughlin, M. Preparation of endothelin antagonist ABT-627. Tetrahedron Lett. 1999, 40, 7175-7178. [CrossRef]

11. Ishihara, K.; Ohara, S.; Yamamoto, H. 3,4,5-trifluorobenzeneboronic acid as an extremely active amidation catalyst. J. Org. Chem. 1996, 61, 4196-4197. [CrossRef] [PubMed]

12. Gangwar, S.; Pauletti, G.; Siahaan, T.; Stella, V.; Borchardt, R. Synthesis of a novel esterase-sensitive cyclic prodrug of a hexapeptide using an (acyloxy)alkoxy promoiety. J. Org. Chem. 1997, 62, 1356-1362. [CrossRef]

13. Rajappan, V.; Hosmane, R. Pentafluorophenol: A superior reagent for condensations in heterocyclic chemistry. Synth. Commun. 1998, 28, 753-764. [CrossRef]

14. Sanchez-Sancho, F.; Mann, E.; Herradon, B. Efficient syntheses of polyannular heterocycles featuring microwave-accelerated Bischler-Napieralski reaction, stereoselective Heck cyclization, and Claisen rearrangement. Synlett 2000, 509-513. [CrossRef]

15. Ragnarsson, U.; Grehn, L. Novel amine chemistry based on DMAP-catalyzed acylation. Acc. Chem. Res. 1998, 31, 494-501. [CrossRef]

16. Zhang, L.; Wang, X.; Wang, J.; Grinberg, N.; Krishnamurthy, D.; Senanayake, C. An improved method of amide synthesis using acyl chlorides. Tetrahedron Lett. 2009, 50, 2964-2966. [CrossRef]

17. Engers, D.; Field, J.; Le, U.; Zhou, Y.; Bolinger, J.; Zamorano, R.; Blobaum, A.; Jones, C.; Jadhav, S.; Weaver, C.; et al. Discovery, Synthesis, and Structure-Activity Relationship Development of a Series of N-(4-Acetamido)phenylpicolinamides as Positive Allosteric Modulators of Metabotropic Glutamate Receptor 4 (mGlu(4)) with CNS Exposure in Rats. J. Med. Chem. 2011, 54, 1106-1110. [CrossRef]

18. Bousfield, T.; Pearce, K.; Nyamini, S.; Angelis-Dimakis, A.; Camp, J. Synthesis of amides from acid chlorides and amines in the bio-based solvent Cyrene (TM). Green Chem. 2019, 21, 3675-3681. [CrossRef]

19. Sayes, M.; Charette, A. Diphenylsilane as a coupling reagent for amide bond formation. Green Chem. 2017, 19, 5060-5064. [CrossRef] 
20. Galvez, A.; Schaack, C.; Noda, H.; Bode, J. Chemoselective Acylation of Primary Amines and Amides with Potassium Acyltrifluoroborates under Acidic Conditions. J. Am. Chem. Soc. 2017, 139, 1826-1829. [CrossRef]

21. Braddock, D.; Lickiss, P.; Rowley, B.; Pugh, D.; Purnomo, T.; Santhakumar, G.; Fussell, S. Tetramethyl Orthosilicate (TMOS) as a Reagent for Direct Amidation of Carboxylic Acids. Org. Lett. 2018, 20, 950-953. [CrossRef] [PubMed]

22. Sabatini, M.; Boulton, L.; Sheppard, T. Borate esters: Simple catalysts for the sustainable synthesis of complex amides. Sci. Adv. 2017, 3. [CrossRef]

23. Schoenberg, A.; Bartoletti, I.; Heck, R.F. Palladium-catalyzed carboalcoxilation of aryl, benzyl and vinylic halides. J. Org. Chem. 1974, 39, 3318-3326. [CrossRef]

24. Schoenberg, A.; Heck, R.F. Palladium-catalyzed amidation of aryl, heterocyclic, and vinylic halides. J. Org. Chem. 1974, 39, 3327-3331. [CrossRef]

25. Schoenberg, A.; Heck, R.F. Palladium-catalyzed formylation of aryl, heterocyclic, and vinylic halides. J. Am. Chem. Soc. 1974, 96, 7761-7765. [CrossRef]

26. Wu, X.; Neumann, H.; Beller, M. Synthesis of Heterocycles via Palladium-Catalyzed Carbonylations. Chem. Rev. 2013, 113, 1-35. [CrossRef] [PubMed]

27. Roy, S.; Gribble, G. Metal-catalyzed amidation. Tetrahedron 2012, 68, 9867-9923. [CrossRef]

28. Wu, X.; Neumann, H.; Beller, M. Palladium-catalyzed carbonylative coupling reactions between Ar-X and carbon nucleophiles. Chem. Soc. Rev. 2011, 40, 4986-5009. [CrossRef] [PubMed]

29. Magano, J.; Dunetz, J. Large-Scale Applications of Transition Metal-Catalyzed Couplings for the Synthesis of Pharmaceuticals. Chem. Rev. 2011, 111, 2177-2250. [CrossRef] [PubMed]

30. Grigg, R.; Mutton, S. Pd-catalysed carbonylations: Versatile technology for discovery and process chemists. Tetrahedron 2010, 66, 5515-5548. [CrossRef]

31. Brennfuhrer, A.; Neumann, H.; Beller, M. Palladium-Catalyzed Carbonylation Reactions of Aryl Halides and Related Compounds. Angew. Chem. Int. Ed. 2009, 48, 4114-4133. [CrossRef] [PubMed]

32. Barnard, C. Palladium-Catalyzed Carbonylation-A Reaction Come of Age. Organometallics 2008, 27, 5402-5422. [CrossRef]

33. Skoda-Foldes, R.; Kollar, L. Synthetic applications of palladium catalysed carbonylation of organic halides. Curr. Org. Chem. 2002, 6, 1097-1119. [CrossRef]

34. Wu, X.; Neumann, H.; Beller, M. Selective Palladium-Catalyzed Aminocarbonylation of Aryl Halides with CO and Ammonia. Chem. Eur. J. 2010, 16, 9750-9753. [CrossRef] [PubMed]

35. Gadge, S.; Bhanage, B. Recent developments in palladium catalysed carbonylation reactions. RSC Adv. 2014, 4, 10367-10389. [CrossRef]

36. Wu, X. Palladium-catalyzed carbonylative transformation of aryl chlorides and aryl tosylates. RSC Adv. 2016, 6, 83831-83837. [CrossRef]

37. Peng, J.; Qi, X.; Wu, X. Recent Achievements in Carbonylation Reactions: A Personal Account. Synlett 2017, $28,175-194$. [CrossRef]

38. Marosvolgyi-Hasko, D.; Lengyel, B.; Tukacs, J.; Kollar, L.; Mika, L. Application of -Valerolactone as an Alternative Biomass-Based Medium for Aminocarbonylation Reactions. ChemPlusChem 2016, 81, 1224-1229. [CrossRef]

39. Marosvolgyi-Hasko, D.; Kegl, T.; Kollar, L. Substituent effects in aminocarbonylation of para-substituted iodobenzenes. Tetrahedron 2016, 72, 7509-7516. [CrossRef]

40. Nagymihaly, Z.; Caturello, N.; Takatsy, A.; Aragay, G.; Kollar, L.; Albuquerque, R.; Csok, Z. Palladium-Mediated Catalysis Leads to Intramolecular Narcissistic Self-Sorting on a Cavitand Platform. J. Org. Chem. 2017, 82, 390-396. [CrossRef]

41. Gergely, M.; Kollar, L. Aminocarbonylation (hydrazinocarbonylation) of iodoalkenes and iodoarenes. Tetrahedron 2017, 73, 838-844. [CrossRef]

42. Gergely, M.; Boros, B.; Kollar, L. High-yielding synthesis of N-triazolyl carboxamides via palladium-catalysed aminocarbonylation. Tetrahedron 2017, 73, 6736-6741. [CrossRef]

43. Mikle, G.; Boros, B.; Kollar, L. Asymmetric aminocarbonylation of iodoalkenes in the presence of alpha-phenylethylamine as an N-nucleophile. Tetrahedron Asymmetry 2017, 28, 1733-1738. [CrossRef]

44. Nagymihaly, Z.; Csok, Z.; Kollar, L. Influence of base additives on the selectivity of palladium-catalysed aminocarbonylation: Highly selective functionalization of a cavitand scaffold. Mol. Catal. 2018, 444, 70-75. [CrossRef]

45. Kollar, L.; Varga, M.; Dornyei, A.; Takacs, A. Functionalisation of the uracil ring via palladium-catalysed aminocarbonylation. Tetrahedron 2019, 75, 4632-4639. [CrossRef]

46. Szoke, G.; Takacs, A.; Kollar, L. Synthesis of Pyridazine Dicarboxamides via Highly Selective Palladium-catalyzed Aminocarbonylation. J. Heterocycl. Chem. 2016, 53, 2020-2024. [CrossRef]

47. Takacs, A.; Marosvolgyi-Hasko, D.; Kabak-Solt, Z.; Damas, L.; Rodrigues, F.; Carrilho, R.; Pineiro, M.; Pereira, M.; Kollar, L. Functionalization of indole at C-5 or C-7 via palladium-catalysed double carbonylation. A facile synthesis of indole ketocarboxamides and carboxamide dimers. Tetrahedron 2016, 72, 247-256. [CrossRef]

48. Szoke, G.; Takacs, A.; Berente, Z.; Petz, A.; Kollar, L. Synthesis of amino-substituted pyridylglyoxylamides via palladium-catalysed aminocarbonylation. Tetrahedron 2016, 72, 3063-3067. [CrossRef]

49. Takacs, A.; Varga, G.; Kardos, J.; Kollar, L. Palladium-catalysed aminocarbonylation of diiodopyridines. Tetrahedron 2017, 73, 2131-2138. [CrossRef] 
50. Cox, R.; Ritson, D.; Dane, T.; Berge, J.; Charmant, J.; Kantacha, A. Room temperature palladium catalysed coupling of acyl chlorides with terminal alkynes. Chem. Commun. 2005, 1037-1039. [CrossRef]

51. Cox, R.J.; Evitt, A.S. Acyl palladium species in synthesis: Single-step synthesis of alpha, beta-unsaturated ketones from acid chlorides. Org. Biomol. Chem. 2007, 5, 229-232. [CrossRef]

52. Gao, B.; Zhang, G.; Zhou, X.; Huang, H. Palladium-catalyzed regiodivergent hydroaminocarbonylation of alkenes to primary amides with ammonium chloride. Chem. Sci. 2018, 9, 380-386. [CrossRef] [PubMed]

53. Willand, N.; Folleas, B.; Boutillon, C.; Verbraeken, L.; Gesquiere, J.; Tartar, A.; Deprez, B. Efficient, two-step synthesis of N-substituted nortropinone derivatives. Tetrahedron Lett. 2007, 48, 5007-5011. [CrossRef]

54. Neufeldt, S.; Jimenez-Oses, G.; Comins, D.; Houk, K. A Twist on Facial Selectivity of Hydride Reductions of Cyclic Ketones: Twist-Boat Conformers in Cyclohexanone, Piperidone, and Tropinone Reactions. J. Org. Chem. 2014, 79, 11609-11618. [CrossRef]

55. Affolter, O.; Baro, A.; Laschat, S.; Fischer, P. Acylation of tropane alkaloids displaying reversed diastereoselectivities under enzymatic versus chemical conditions. Zeitschr. Naturforsch. Sect. B J. Chem. Sci. 2007, 62, 82-92. [CrossRef]

56. Wang, Z.; Hou, Z.; Yao, S.; Lin, M.; Song, H. A new and recyclable system based on tropin ionic liquids for resolution of several racemic amino acids. Anal. Chim. Acta. 2017, 960, 81-89. [CrossRef] [PubMed]

57. Lyapkalo, I.; Hogermeier, J.; Reissig, H. Synthesis of new tropinone derivatives by palladium-catalyzed couplings of 8 azabicyclo[3.2.1] oct-2-enyl nonatlates. Tetrahedron 2004, 60, 7721-7729. [CrossRef]

58. Cheng, J.; Moore, Z.; Stevens, E.; Trudell, M. Stereoselective syntheses of the three isomers of ethylene glycol bis(tropane-3carboxylate). J. Org. Chem. 2002, 67, 5433-5436. [CrossRef]

59. Ghosh, S.; Kinney, W.; Gauthier, D.; Lawson, E.; Hudlicky, T.; Maryanoff, B. Convenient preparation of aryl-substituted nortropanes by Suzuki-Miyaura methodology. Can. J. Chem. 2006, 84, 555-560. [CrossRef]

60. Horvath, L.; Berente, Z.; Kollar, L. High-yielding aminocarbonylation of 3-iodo-2-tropene by using amino acid esters as Nnucleophiles. Lett. Org. Chem. 2007, 4, 236-238. [CrossRef]

61. Nocquet, P.; Opatz, T. Total Synthesis of (+/-)-Scopolamine: Challenges of the Tropane Ring. Eur. J. Org. Chem. 2016, 2016, 1156-1164. [CrossRef]

62. Fodor, G.; Nádor, K. The stereochemistry of tropane alkaloids. Part I. The configuration of tropine and $\psi$-tropine. J. Chem. Soc. 1953, 721-723. [CrossRef]

63. Bollinger, S.; Hubner, H.; Heinenmann, F.; Meyer, K.; Gmeiner, P. Novel Pyridylmethylamines as Highly Selective 5-HT1A Superagonists. J. Med. Chem. 2010, 53, 7167-7179. [CrossRef]

64. Kohnen-Johannsen, K.; Kayser, O. Tropane Alkaloids: Chemistry, Pharmacology, Biosynthesis and Production. Molecules 2019, 24, 796. [CrossRef]

65. Skoda-Foldes, R.; Kollar, L. Palladium-Catalyzed Aminocarbonylation of Iodoalkenes and Iodoarenes. Lett. Org. Chem. 2010, 7 , 621-633. [CrossRef]

66. Kiss, M.; Takacs, A.; Kollar, L. Highly Selective Synthesis of Carboxamides via Transition Metal Catalysed Aminocarbonylation. Curr. Green Chem. 2015, 2, 319-338. [CrossRef]

67. Amatore, C.; Jutand, A.; Mbarki, M. Evidence of the formation of zerovalentpalladium from $\mathrm{Pd}(\mathrm{OAc})_{2}$ and triphenylphosphine. Organometallics 1992, 11, 3009-3013. [CrossRef]

68. Amatore, C.; Carre, E.; Jutand, A.; Mbarki, M.; Meyer, G. Evidence for the Ligation of Palladium(0) Complexes by Acetate Ions: Consequences on the Mechanism of Their Oxidative Addition with Phenyl Iodide and $\mathrm{PhPd}(\mathrm{OAc})\left(\mathrm{PPh}_{3}\right)_{2}$ as Intermediate in the Heck Reaction. Organometallics 1995, 14, 5605-5614. [CrossRef]

69. Csakai, Z.; Skoda-Foldes, R.; Kollar, L. NMR investigation of $\mathrm{Pd}(\mathrm{II})-\mathrm{Pd}(0)$ reduction in the presence of mono- and ditertiary phosphines. Inorg. Chim. Acta 1999, 286, 93-97. [CrossRef]

70. Takacs, A.; Farkas, R.; Kollar, L. High-yielding synthesis of 2-arylacrylamides via homogeneous catalytic aminocarbonylation of $\alpha$-iodostyrene and $\alpha, \alpha^{\prime}$-diiodo-1,4-divinylbenzene. Tetrahedron 2008, 64, 61-66. [CrossRef]

71. Takacs, A.; Acs, P.; Berente, Z.; Wolfling, J.; Schneider, G.; Kollar, L. Novel 13 beta- and 13 alpha-D-homo steroids: 17acarboxamido-D-homoestra-1,3,5(10),17-tetraene derivatives via palladium-catalyzed aminocarbonylations. Steroids 2010, 75, 1075-1081. [CrossRef] [PubMed]

72. Kollar, L.; Takacs, A. Novel synthesis of 3-carboxamidolactam derivatives via palladium-catalysed aminocarbonylation. Tetrahedron 2018, 74, 6116-6128. [CrossRef]

73. Muller, E.; Peczely, G.; Skoda-Foldes, R.; Takacs, E.; Kokotos, G.; Bellis, E.; Kollar, L. Homogeneous catalytic aminocarbonylation of iodoalkenes and iodobenzene with amino acid esters under conventional conditions and in ionic liquids. Tetrahedron 2005, 61, 797-802. [CrossRef]

74. Kiss, M.; Maho, S.; Boddi, K.; Boros, B.; Kollar, L. Palladium-catalyzed diaminocarbonylation: Synthesis of androstene dimers containing 3,3'- or 17,17'-dicarboxamide spacers. Monatsh. Chem. 2015, 146, 357-364. [CrossRef]

75. Barton, D.; O'Brien, R.; Sternhell, S. A new reaction of hydrazones. J. Chem. Soc. 1962, 470-476. [CrossRef]

76. Barton, D.; Bashiardes, G.; Fourrey, J. An Improved Preparation of Vinyl Iodides. Tetrahedron Lett. 1983, 24, 1605-1608. [CrossRef] 